\title{
Memorizing While Walking: Increase in Dual-Task Costs From Young Adulthood to Old Age
}

\author{
Ulman Lindenberger, Michael Marsiske, and Paul B. Baltes \\ Max Planck Institute for Human Development
}

\begin{abstract}
The dual task of memorizing word lists while walking was predicted to become more difficult with age because balance and gait are in greater need of "attentional resources." Forty-seven young (ages 20-30 years), 45 middle-aged (40-50), and 48 old $(60-70)$ adults were trained to criterion in a mnemonic technique and instructed to walk quickly and accurately on 2 narrow tracks of different path complexity. Then, participants encoded the word lists while sitting, standing, or walking on either track; likewise, speed and accuracy of walking performance were assessed with and without concurrent memory encoding. Dual-task costs increased with age in both domains; relative to young adults, the effect size of the overall increase was 0.98 standard deviation units for middle-aged and 1.47 standard deviation units for old adults. It is argued that sensory and motor aspects of behavior are increasingly in need of cognitive control with advancing age.
\end{abstract}

The main purpose of this study was to test the prediction that the simultaneous execution of a challenging locomotion task-walking on a narrow track-and a memory task-memorizing a list of words--becomes increasingly difficult with advancing age. This prediction was motivated by the hypothesis that sensorimotor performance is increasingly in need of "attentional resources" (Craik \& Byrd, 1982) with advancing age (Brown \& Woollacott, 1998; Teasdale, Lajoie, Bard, Fleury, \& Courtemanche, 1993; cf. Welford, 1958, pp. 186-187). As an illustration, imagine 20-yearold and 70-year-old pedestrians crossing a lively street corner. Because of losses in motor and sensory functioning, 70-year-old individuals will need, on average, to exert more attention to successfully perform this task than will 20-year-old individuals. As a consequence, one may hypothesize that they will be more likely than young adults to interrupt a conversation with another person while crossing the street.

Ulman Lindenberger, Michael Marsiske, and Paul B. Baltes, Max Planck Institute for Human Development, Berlin, Germany. Michael Marsiske is now at the Institute on Aging, University of Florida.

The present research was conducted in the context of the projects Memory and Intelligence in Development (MIND) and Selection, Optimization, and Compensation (SOC) at the Max Planck Institute for Human Development. We acknowledge the invaluable advice of Benno Nigg and Klaus Scherer, as well as helpful discussions with Peter Frensch, Alexandra Freund, Daniela Jopp, Jutta Kray, Karen $\mathrm{Li}$, Shu-Chen $\mathrm{Li}$, and Tania Singer. In addition, we thank Werner Scholtysik for his expert help in setting up the walking track; Matthias Stroux and Manfred Weilandt for programming assistance; Annette Rentz-Lühning, Daniela Jopp, Ulrike Bär, Mike Kortsch, Tania Singer, and Manfred Weilandt for data collection; and the research participants for their willingness to participate in a demanding study.

Correspondence concerning this article should be addressed to Ulman Lindenberger, who is now at the Developmental Psychology Unit, Building 1, Saarland University, Im Stadtwald, D-66123 Saarbrücken, Germany, or to Paul B. Baltes, Max Planck Institute for Human Development, Lentzeallee 94, D-14195 Berlin, Germany. Electronic mail may be sent to lindenberger@mx.uni-saarland.de or to sekbaltes@mpib-berlin.mpg.de.
As suggested by this illustration, and informed by studies reporting a strong correlational link between sensorimotor and cognitive performance in old age (Anstey, Lord, \& Williams, 1997; Anstey, Stankov, \& Lord, 1993; Baltes \& Lindenberger, 1997; Lindenberger \& Baltes, 1994, 1997), we used the dual-task paradigm of cognitive psychology to test the prediction that walking becomes cognitively more demanding with advancing age (cf. Chen et al., 1996; Mulder, Berndt, Pauwels, \& Nienhuis, 1993) Specifically, we examined whether old adults (60-70 years of age) would exhibit greater decrements in performance than young adults (20-30 years of age) when they were simultaneously walking on a narrow track and memorizing lists of words by use of a mnemonic aid. A group of middle-aged adults (40-50 years of age) was included to explore the possible onset of such aginginduced increments in dual-task costs (DTCs).

Our decision to combine a walking task with an episodic memory task was guided by two motives. First, we assumed that episodic memory and locomotion, the functional domains represented by the two tasks, possess high degrees of ecological validity and undergo substantial decrements with advancing age. Losses in episodic memory (Baltes \& Kliegl, 1992; Light, 1996; Zacks, Hasher, \& Li, 2000) and locomotion (Winter, 1991) are ubiquitous with aging. At the same time, both functional domains are of central importance for older persons' everyday competence and well-being (Marsiske, Klumb, \& Baltes, 1997). The second reason was more methodological in kind. By choosing two relatively challenging tasks, we wanted to make sure that a possible absence of DTCs, or of age differences in DTCs, could not be due to insufficient task difficulty.

In the following, we first provide a brief sketch of the cognitive literature on adult age differences in DTCs (see also McDowd \& Shaw, 2000; Ribaupierre \& Ludwig, 2000). Then, we review the relevant literature on adult age differences in postural control, with a focus on standing and walking. Finally, we mention methodological problems in the age-comparative study of DTC and provide an overview of this study. 


\section{Adult Age Differences in DTCs: A Tentative Taxonomy}

Much of the age-comparative work using the dual-task paradigm has been informed by the resource metaphor of attentiondemanding processes (e.g., Craik \& Byrd, 1982). Based on that metaphor, the general expectation is that old adults show greater DTCs than young adults because they have less attentional resources to simultaneously execute two or more tasks instead of one. The widespread existence of positive age differences in the absolute magnitude of DTCs seems to support this contention.

However, ever since Brinley (1965), it has been noted that age comparisons using a proportional metric often yield a different picture. For instance, McDowd and Craik (1987) plotted old adults' reaction times as a function of young adults and found that all data points were well fit by one linear function, regardless of whether they represented performance under single-task or dualtask conditions. In other words, age differences in DTCs were present with difference scores but absent when a relative (proportional) metric was adopted. A parsimonious explanation for this pattern of results is that age differences under multiple-task conditions are the additive outcome of age differences in each of the single tasks (cf. Hartley, 1992).

More recently, though, a growing number of studies have reported disproportional age differences in DTCs under certain task conditions (Korteling, 1993; Kray \& Lindenberger, 2000; Ribaupierre \& Ludwig, 2000; Salthouse, Hambrick, Lukas, \& Dell, 1996; Tsang \& Shaner, 1998). Apparently, then, age differences in DTCs vary as a function of task conditions. As a consequence, the prediction of age differences in DTCs has to be complemented by assumptions about processing mechanisms for each of the two tasks, both performed in isolation and performed together, as well as the expected age differences in these mechanisms (cf. McDowd \& Shaw, 2000).

A first step toward a more process-oriented understanding of age differences in DTCs is to list task characteristics that appear to favor the presence of large (i.e., disproportional) age differences in DTCs (cf. Ribaupierre \& Ludwig, 2000). Generally, it seems that age differences in DTC are large (a) when the two tasks share the same stimulus modality, working memory representation, or response modality, especially if the stimulus-response mappings of the two tasks overlap in incompatible ways (Korteling, 1993; Kray \& Lindenberger, 2000; Tsang \& Shaner, 1998; cf. Navon \& Gopher, 1979; Wickens, 1984); (b) when information regarding the identity and sequencing of the two tasks has to be maintained and coordinated without the aid of external cues (e.g., Kray \& Lindenberger, 2000); and (c) when one or more of the constituent tasks themselves impose high demands on cognitive control processes such as focusing attention, scheduling and planning, and updating and checking, as well as coding contextual representations (e.g., Duncan, Emslie, \& Williams, 1996; Salthouse et al., 1996).

On the basis of this list, it seems that age differences in DTCs tend to be magnified when combining the two tasks induces a marked increase in the need for cognitive control and supervision (Shallice \& Burgess, 1993). This is the case, for instance, when two tasks critically depend on the same processes or cognitive components. In such situations, processing of the two tasks must be regulated to avoid structural interference and cross talk (cf. Kinsbourne \& Hicks, 1978; Wickens, 1984). Hence, age-based increments in DTCs are especially likely to be observed whenever the processing demands of the constituent tasks become more similar to each other with advancing age. It is assumed that the two tasks combined in this study meet this criterion because postural control becomes increasingly attention-demanding with advancing age. Specifically, the available literature (as described below) indicates that postural aspects of behavior in general, and of walking in particular, impose greater cognitive control demands on old than on young adults. Given that skilled episodic memory performance requires cognitive control in both young and old adults, the simultaneous execution of these two tasks should be associated with a greater degree of processing overlap, or structural interference, with advancing age. This expectation is reinforced by more specific evidence demonstrating that episodic memory with the method of loci (MOL; Baddeley \& Lieberman, 1980; Lindenberger, Kliegl, \& Baltes, 1992) as well as posture control (Kerr, Condon, \& McDonald, 1985) both involve visuospatial processing, and that the dependency of locomotion on visual information appears to increase with age (Anderson, Nienhuis, Mulder, \& Hulstijn, 1998). For both of these reasons, we predicted greater processing incompatibility between the two tasks and, as a result, an increase in DTCs from young adulthood to old age.

\section{Adult Age Differences in Postural Stability and Locomotion: The Importance of Cognitive Control}

The high prevalence of falls in the elderly is probably the most dramatic symptom of the increasing difficulty of avoiding maladaptive postural sway while standing or walking that is experienced by aging individuals (Blake et al., 1988). The maintenance of postural stability while standing or walking requires the continuous coordination and integration of visual, proprioceptive, and vestibular sensory information in several areas of the brain, including the cerebellum, the brainstem, the basal ganglia, and sensorimotor cortex (Woollacott \& Jensen, 1996), as well as the execution of the corresponding movements by the limb and trunk muscles that receive impulses from the spinal cord and peripheral nerves.

There is general agreement that the cognitive control demands for standing and walking increase with age (for a summary, see Brown \& Woollacott, 1998). Normal aging appears to negatively affect all stages of the postural control system, resulting in less reliable sensory information, less accurate integration, and less effective postural control (Alexander, 1994; Brown \& Woollacott, 1998; Stelmach, Teasdale, DiFabio, \& Phillips, 1989; for relevant neurophysiological evidence in rodents, see Spengler, Godde, \& Dinse, 1995). In addition to more peripheral changes in sensory and motor domains, age-comparative analyses of micropostural adjustments have demonstrated that cognitive control processes contribute significantly to age differences in maintaining a stable upright stance (Brown, Shumway-Cook, \& Woollacott, 1999; Lajoie, Teasdale, Bard, \& Fleury, 1996; Maylor, Allison, \& Wing, in press; Maylor \& Wing, 1996; Shumway-Cook, Woollacott, Kerns, \& Baldwin, 1997; Stelmach, Zelaznik, \& Lowe, 1990; Teasdale et al., 1992; Teasdale, Bard, LaRue, \& Fleury, 1993; Teasdale, Stelmach, \& Breunig, 1991). A smaller number of age-comparative studies in the domain of walking show similar results (Chen, 
Ashton-Miller, Alexander, \& Schultz, 1994; Chen et al., 1996; Lajoie et al., 1996; Mulder et al., 1993).

With respect to the dual-task paradigm, many investigations have combined cognitive tasks with motor tasks involving isolated arm movements (e.g., Baddeley \& Lieberman, 1980; Korteling, 1993). However, cognitive tasks have been combined less often with sensorimotor tasks imposing greater demands on posture, such as standing or walking. In a classical study of young adults, Kerr, Condon, and McDonald (1985) found that performance on a spatial working memory task decreased when participants were asked to perform the task while maintaining a difficult posture as compared with their performance under seated conditions.

Only a few studies have used the dual-task paradigm to examine age differences in the cognitive demands of posture control. Specifically, research interest has focused on two motor activities: (a) standing (Brown et al., 1999; Maylor et al., in press; Maylor \& Wing, 1996; Shumway-Cook et al., 1997) and (b) walking (Chen et al., 1996; Mulder et al., 1993). The results of these studies suggest that the magnitude of DTCs associated with standing on a solid platform are generally small, with age differences in DTCs often being restricted to the degree of postural sway (Maylor \& Wing, 1996; Shumway-Cook et al., 1997). Age differences in DTCs increase when the platform is compliant or moving, most likely because cognitive control demands are higher under such task conditions (Brown et al., 1999). Finally, two studies have found sizable age differences in DTCs when individuals were asked to walk while performing a cognitive task (Chen et al,, 1996; Mulder et al., 1993). This is consistent with the notion that "sitting, standing, and walking represent a postural hierarchy with respect to attentional demands" (Shumway-Cook et al., 1997, p. M238).

To summarize, normal aging is associated with decrements in the reliability and automaticity of elementary and coordinative processes involved in posture control. The degree to which these decrements are associated with a concomitant increase in cognitive demands appears to depend, among other things, on task difficulty. The present investigation extends these earlier observations by using a new combination of task domains with a high degree of ecological validity (e.g., walking and memorizing). In addition, it examines the ontogenetic onset of adult age differences in DTCs for this combination of tasks by including a group of middle-aged adults. Finally, a number of methodological refinements are introduced to enhance the interpretability of results.

\section{This Study: Methodological Considerations}

In this study, research participants of different ages were asked either to memorize a list of words using the MOL (Bower, 1970), to walk on a narrow track as fast and as accurately as possible, or to perform both tasks simultaneously. The dual-task manipulation was restricted to the encoding phase of the memory task; thus, the crucial question was whether performance costs induced by the simultaneous memory encoding and walking increased with age. As others have noted before (e.g., Craik \& Jennings, 1992; Kramer, Larish, \& Strayer, 1995; McDowd \& Shaw, 2000; Salthouse, 1988), the quasi-experimental nature of this question poses difficult methodological problems. For instance, one would like to know whether age differences under dual-task conditions are greater than expected on the basis of age differences in single-task performance. For this purpose, it is advisable (a) to administer single and dual tasks at overlapping levels of difficulty (cf. Kramer et al., 1995), or (b) to equate young and old participants on single-task performance by means of adaptive testing procedures or differential task exposure (Kliegl \& Lindenberger, 1993). In addition, it is generally more appropriate (c) to express DTCs in relative, rather than absolute terms (e.g., as percentage of loss relative to single-task performance; cf. Baddeley, Della Sala, Gray, Papagno, \& Spinnler, 1997).

In the present study, we used a combination of all three strategies. With respect to the memory task, research participants were trained in serial recall with the MOL until they reached a prespecified criterion level. This procedure has several advantages. First, the instruction in and acquisition of a mnemonic device such as the MOL greatly enhances the degree of experimental control over memory-related processing (Baltes \& Kliegl, 1992). Second, in comparison to most other age-comparative research on DTCs, we expected that young and old adults would be operating at more similar levels of memory performance after having been trained to criterion, which would enhance the interpretability of age differences in DTCs.

With respect to walking performance, research participants were asked to walk as accurately and quickly as possible on two narrow tracks that differed from each other in path complexity (see Figure 1). We assumed that the topologically more complex, "aperiodic" track would require more cognitive processing than the less complex, "oval" track and hence be associated with larger DTCs in memorizing and with larger age differences in memorizing DTCs. Second, about half of the research participants in each age group received four sessions of walking practice prior to the critical assessment of single- and dual-task performance. Walking practice involved trial-by-trial feedback regarding both speed and accuracy parameters of walking and was expected to result in better (i.e., faster and more accurate) walking performance, lower cognitive demands, and a reduction in DTCs. Thus, instead of trying to equate individuals of different ages on single-task proficiency (as was the case for the memory task), the difficulty and complexity of the walking task were varied by two levels of single-task practice and track complexity.

A final methodological consideration concerns the assessment of the processing dynamics of dual-task performance (cf. Maylor et al., in press). Research participants of all ages were expected to optimize performance under dual-task conditions by shifts of attention between the memory and the walking tasks. On the basis of previous findings (Baddeley \& Lieberman, 1980; Bower, 1970; Bugelski, 1970; Lea, 1975; Lindenberger et al., 1992), we assumed that the formation of associations between to-be-learned words and locations, most likely by means of mental imagery, would be the most effortful cognitive operation in the context of the memory task. Therefore, we expected that research participants of all ages, and perhaps especially old adults, would show a pronounced decrement in parameters of walking performance, such as step frequency, during the association-formation phase of MOL performance, compared with the initial auditory word comprehension phase. 

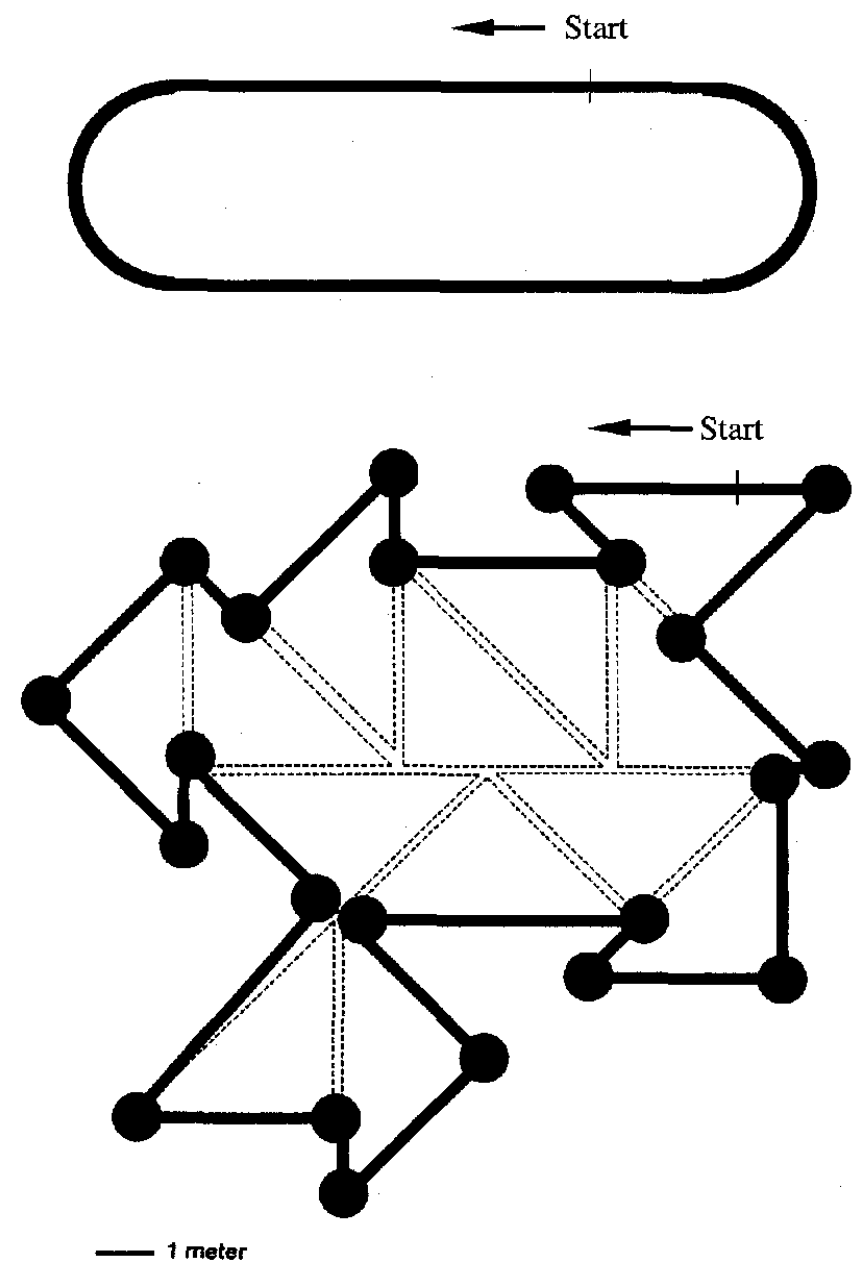

Figure 1. Schematic drawing of the oval and aperiodic walking tracks used in the present study. Triangles within the aperiodic track were not physically present but are meant to illustrate the design of this track (i.e., aperiodic monohedral tiling with an isosceles triangle).

\section{Overview of Study and Data Analysis}

\section{Study Design}

The present study consisted of five major parts (see Table 1): (a) two pretest sessions; (b) memory training with the MOL, with an average of 5.5 sessions of instruction and training to criterion; (c) either four or zero sessions of walking practice depending on experimental condition; (d) two sessions of posttest, including the critical experimental conditions of memorizing with and without concurrent walking performance, and of walking with and without concurrent memorizing; and (e) a final session with debriefing and additional questionnaires.

Depending on the absence versus presence of walking practice, the experiment consisted of an average of 14.5 or 10.5 sessions, respectively. Sessions were scheduled twice a week, with two exceptions: (a) Participants who did not receive walking practice received no contact for about 2 to 3 weeks, that is, for about the time it took to administer the four practice sessions to participants in the walking-practice condition, and (b) the debriefing session took place several weeks after the second posttest session. ${ }^{1}$

\section{General Features of Data Analysis}

The present study involved noncontiguous age groups of young, middle-aged, and old adults. Therefore, age was treated as a factor with three levels, not as a continuous variable. For all age-group comparisons, we defined two orthogonal contrasts reflecting the assumption that young adults constitute a reference group for the two older age groups. The first contrast tested whether middleaged and old adults differed from young adults, and the second whether middle-aged adults differed from old adults. Data were routinely checked for variance (Cochran's C) or variancecovariance (Box's $M$ ) homogeneity when performing simple or repeated measures analyses of variance (ANOVAs), respectively; unless stated otherwise, the null hypothesis of variance homogeneity could be retained. Alpha levels were set to .01 , and values for partial $\eta^{2}$ were reported to indicate effect size. In the case of univariate $F$ tests with $1 d f, \eta^{2}$ corresponds to the square of the point-biserial correlation between the dependent variable and the two levels of the independent variable (cf. Richardson, 1996).

\section{Method}

\section{Sample}

Research participants were recruited through advertisements in local newspapers and were paid DM 20 (Euro 10 , or approximately $\$ 10$ ) per session. Of the 177 adults who initiated participation in this experiment, 140 completed the entire experiment: 47 young adults (mean age $=24$ years, range $=20-30$ years, $S D=3.2$ years), 45 middle-aged adults (mean age $=45$ years, range $=40-50$ years, $S D=3.3$ years), and 48 old adults (mean age $=65$ years, range $=60-70$ years, $S D=3.1$ years). Reasons for sample attrition are listed at the beginning of the Results section. The 140 individuals who participated in the entire experiment constituted the effective sample of this study.

To document the age typicality of the samples, Table 2 displays the average scores of young, middle-aged, and old individuals on Set II of the Raven Advanced Progressive Matrices test (Raven, Court, \& Raven, 1983) as well as their average scores on a vacabulary test (described below; cf. Wechsler, 1982). The test by age group interaction was significant, young versus middle-aged and old, $F(1,137)=181.64, M S E=16.91, p<.01$, $\eta^{2}=.36$, or marginally significant, middle-aged versus old, $F(1$, $137)=6.05, M S E=16.91, p=.015, \eta^{2}=.04$. Performance on the Raven Advanced Progressive Matrices test decreased as a function of age, young versus middle-aged and old adults, $F(1,137)=99.18, M S E=26.69, p<$ $.01, \eta^{2}=.42$; middle-aged versus old adults, $F(1,137)=12.94$, $M S E=26.69, p<.01, \eta^{2}=.08$. In contrast, performance on the vocabulary test did not vary significantly as a function of age group, young versus middle-aged and old adults, $F(1,137)=0.02, M S E=17.71, p>$ .80 ; middle-aged versus old adults, $F(1,137)=1.04, M S E=17.71, p>$ 30. Thus, the typical developmental dissociation between decrements in the fluid mechanics and maintenance in the crystallized pragmatics of intelligence was observed (Baltes, 1987; Horn, 1989). It can be concluded

\footnotetext{
1 The present study also addressed a few other issues, such as the relationship between training gains and efficacy beliefs (e.g., Jopp, 1996). Measures related to these other issues were administered during the two pretest sessions, the second posttest session, and the final debriefing session and did not compromise the experimental design of the study.
} 
Table 1

Design of the Study

\begin{tabular}{|c|c|c|c|}
\hline \multicolumn{4}{|c|}{ Study phase } \\
\hline Pretest & Serial recall training & Walking practice & Posttest \\
\hline $\begin{array}{l}\text { First pretest session } \\
\text { Demographics } \\
\text { Raven, Vocabulary } \\
\text { Serial recall: baseline } \\
\text { under seated encoding } \\
\text { conditions } \\
\text { Second pretest session } \\
\text { Walking without MOL } \\
\text { Auditory comprehension } \\
\text { (seated, standing, walking) }\end{array}$ & $\begin{array}{l}\text { Instruction in MOL (Session 3) } \\
\text { Training to criterion with MOL } \\
\text { (seated, up to nine sessions) }\end{array}$ & $\begin{array}{l}\text { Half of the participants } \\
\text { in each age group received } \\
\text { four sessions of walking } \\
\text { practice; the remaining } \\
\text { participants served as a } \\
\text { control group }\end{array}$ & $\begin{array}{l}\text { First posttest session } \\
\text { MOL under seated EC } \\
\text { MOL under standing EC } \\
\text { MOL under walking EC } \\
\text { Walking without MOL } \\
\text { Second posttest session } \\
\text { Walking without MOL } \\
\text { Auditory comprehension } \\
\text { (seated, standing, walking) }\end{array}$ \\
\hline
\end{tabular}

Note. MOL $=$ method of loci; $\mathrm{EC}=$ encoding condition; Raven $=$ Raven Advanced Progressive Matrices test (Raven, Court, \& Raven, 1983); Vocabulary $=$ short form of the German version of the Wechsler Vocabulary test (Lindenberger, Mayr, \& Kliegl, 1993; cf. Wechsler, 1982). Three age groups participated in the experiment: young ( $n=47$, age range $=20-30$ years), middle-aged $(n=45$, age range $=40-50$ years), and old $(n=48$, age range $=60-70$ years). Walking performance was varied at two levels of complexity (see Figure 1). Auditory comprehension conditions at pre- and posttest as well as the experimental conditions of the first posttest session, which served to assess walking and memorizing performance under single- and dual-task conditions, were counterbalanced across subjects within age groups.

that the present samples yield a good approximation of population age gradients in cognitive functioning.

\section{Apparatus}

Five Macintosh SE/30s equipped with closed headphones were used for presentation of verbal stimuli and data entry in the context of memory performance at pretest and during training and for the collection of biographical background information at pretest. Two Macintosh 7100/66s PowerPCs equipped with an infrared sound transmitter, closed headphones, and a National Instruments NB-MIO-16 multifunction I/O board with millisecond timer, provided by National Instruments Germany $\mathrm{GmbH}$, Munich, were used for presentation of verbal stimuli and data entry in the context of memory performance at posttest and for the assessment of walking performance at pretest and posttest. One of the two PowerPCs was used for the oval track, and the other for the aperiodic track. The assessment of walking performance was based on special hardware that we designed for the purpose for this study (see below).

\section{Materials: Memory Performance With the MOL}

The memory task consisted of serial recall of 16-item word lists using the MOL (Bower, 1970). Eight hundred eighty highly imageable, highly concrete nouns (e.g., Baltes \& Kliegl, 1992; Kliegl, Smith, \& Baltes, 1989)

Table 2

Cognitive Characteristics of the Sample as a Function of Age Group

\begin{tabular}{|c|c|c|c|c|c|c|}
\hline \multirow[b]{2}{*}{ Task } & \multicolumn{2}{|c|}{$\begin{array}{c}\text { Young } \\
(n=47)\end{array}$} & \multicolumn{2}{|c|}{$\begin{array}{c}\text { Middle-aged } \\
(n=45)\end{array}$} & \multicolumn{2}{|c|}{$\begin{array}{c}\text { Old } \\
(n=48)\end{array}$} \\
\hline & $M$ & $S D$ & $M$ & $S D$ & $M$ & $S D$ \\
\hline Raven & 20.7 & 5.8 & 13.4 & 5.2 & 9.5 & 4.5 \\
\hline Vocabulary & 21.2 & 4.4 & 21.6 & 4.0 & 20.7 & 4.2 \\
\hline
\end{tabular}

Note. Raven $=$ Raven Advanced Progressive Matrices test (Raven, Court, \& Raven, 1983); Vocabulary = short form of the German version of the Wechsler Vocabulary test (Lindenberger, Mayr, \& Kliegl, 1993; cf. Wechsler, 1982). recorded by a male speaker served as stimuli. Sixteen Berlin landmarks served as implicit location cues during encoding and retrieval after instruction in the MOL (i.e., these cues were not actually presented during encoding or retrieval but were learned by the research participants early in the memory training).

Stimuli were presented auditorily only, using closed headphones, at a rate of $10 \mathrm{~s}$ per word. After the presentation of the last word of a list, research participants had to recall the list in forward serial order. Recall was untimed and visually cued by presenting Arabic numerals corresponding to the serial position of the to-be-remembered word on the computer screen (e.g., "1."). For each serial position, participants responded by typing the first three letters of the to-be-remembered word on the keyboard, making corrections with the backspace key if necessary, and hitting the return key thereafter. After the return key was hit, the digit cue for the next serial position (e.g., "2.") appeared on the screen, and corrections for the earlier position were no longer possible. Participants were instructed to hit the return key directly if they were sure that they did not remember a word at a particular landmark location. During training in the use of the MOL, participants were given accuracy feedback for the list that they just had completed after having responded to the last word of that list. Feedback included overall accuracy (e.g., "10 words correct") and an item-by-item display of recall performance.

With regard to distribution of to-be-learned words over lists, an initial set of 16-item lists was drawn without replacement from the total pool of 880 words. Specifically, stimuli were randomly distributed over lists with the following constraints: (a) Words with identical initial three letters (e.g., crab and crane) were not allowed within the same list, and (b) words judged to be very similar (e.g., king, emperor) were not allowed within the same list. The resulting 55 lists were distributed over sessions in the following manner: (a) memory performance prior to instruction in the MOL (first pretest session, 2 lists), (b) auditory comprehension at pretest under four experimental conditions with 2 lists per condition (i.e., sitting, standing, walking the oval track, walking the aperiodic track; 8 lists), (c) initial instruction session with the MOL (4 lists), (d) the first three training sessions with the MOL with 8 lists per training session (24 lists), (e) memory performance at posttest under four experimental conditions with 2 lists per experimental condition plus 1 initial warm-up list ( 9 lists), and (f) auditory comprehension at posttest under four experimental conditions with 2 lists per condition ( 8 lists). 
Given that memory training with the MOL lasted for a maximum of 10 , rather than 3 , sessions, the maximum number of to-be-leamed words in this study $(N=1,776)$ exceeded the number of available words $(N=880)$. Therefore, an additional set of 56 lists was constructed by drawing additional lists from the total pool of 608 words that had been presented up to the 3rd session of training. The lists in Training Sessions 4-10 followed the same constraints as the other lists (i.e., no ambiguous word beginnings and replacement of high-similarity words). In addition, to avoid interference effects, we ensured that a given word did not appear during the preceding 2 training sessions. Note that earlier findings with the MOL indicate that proactive interference is most pronounced within sessions and rarely extends over more than one session (Kliegl \& Lindenberger, 1993).

The same random distribution of lists over sessions was used for all research participants. Likewise, the order of words within lists was identical across participants.

\section{Materials: Walking Tracks}

In order to assess walking performance at two levels of difficulty and cognitive demand, two narrow tracks of different path complexity were used in this study. The shape of the track with low path complexity (descriptively referred to as the oval track) consisted of two parallel lines with a length of $9 \mathrm{~m}$ connected at both ends by half circles with a diameter of $1.5 \mathrm{~m}$. The shape of the track with high path complexity was based on the mathematics of tiling (Grünbaum \& Shephard, 1987). Specifically, the shape of this path was obtained by monohedral aperiodic tiling of an isosceles triangle (cf. Grünbaum \& Shephard, 1987; see Figure 1). Compared with the oval track, the aperiodic track contained a total of 22 turning points of varying angles instead of 2 curves of equal radius, and 21 straight sections of shorter and more variable lengths compared with 2 straight sections with a length of $9 \mathrm{~m}$. Also, as a direct consequence of the aperiodicity of the tiling procedure, the topology of the aperiodic track contained little rhythmicity or repetition.

The width of the straight sections of both tracks was $19 \mathrm{~cm}$, the width of the curved sections of the oval track varied between 20 and $35 \mathrm{~cm}$, and the diameter of the circular turning points of the aperiodic track was $42 \mathrm{~cm}$. The precise shape of the tracks was defined by aluminum tape and was clearly discernible for all participants.

An electronic device was used to obtain detailed information on a variety of parameters of walking performance such as walking speed, walking accuracy, and step frequency. The main features of this device were (a) that the walking tracks were electrically conductive, (b) that the participants' right and left shoe soles were differentially conductive, and (c) that conductivity information from various channels was recorded by the multifunction I/O board to assess steps and missteps (i.e., steps outside of the aluminum-taped surface of the track) as well as walking speed and walking distance. $^{2}$

\section{Materials: Dual-Task Conditions}

At the first posttest session-that is, after training to criterion in the $\mathrm{MOL}$ and subsequent walking-practice or no-intervention control conditions-research participants performed the MOL under seated, standing, and walking conditions. Verbal stimuli were presented using the same headphones as for MOL, training. In contrast to MOL training, however, the headphones were connected not directly to the computer but rather to an infrared receiver attached to the participants' clothing (e.g., belt, pocket). The corresponding transmitters were connected through the multifunction I/O board to the Macintosh computers that were used to measure walkingrelated performance. Thus, when a participant was walking on a given track under dual-task conditions, the same computer was used to record walking performance and to emit the to-be-learned words to the participant. The corresponding sound signals were clamped, fed into the multifunction I/O board, and projected onto the same time axis as the walking-related information.
At the end of the encoding phase (i.e., $10 \mathrm{~s}$ after the last word), participants under dual-task conditions heard a signal, stopped walking the track, went to the computer, sat down, and initiated the recall phase in the same manner as during MOL training. The distance between the point on the track at which participants happened to be at the end of the encoding phase and the computer was variable but not systematically related to individual differences in walking performance.

At posttest, the identical apparatus (i.e., computer and headphones with infrared equipment) was used under single-task (i.e., seated or standing) conditions, except that no walking-related information was recorded. Under seated encoding conditions, participants sat in a chair in the middle of the room. Under standing encoding conditions, they stood next to the chair in an upright position but without any external support (e.g., without touching the chair). At the end of the encoding phase (i.e., $10 \mathrm{~s}$ after they had heard the last word), participants went to the computer, sat down, and initiated the recall phase. The distance in space between the point at which participants stood or sat during the encoding phase and the computer used for response collection was $8 \mathrm{~m}$, which was about equal to the average of the distances between the computer and the different track locations at which trials ended under dual-task (i.e., walking) conditions.

\section{Procedure}

Testing sites. The study was carried out at the Max Planck Institute for Human Development. The first pretest session and all sessions involving instruction and training with the MOL took place in a sound-resistant laboratory and were conducted in groups of 4 to 5 research participants. The remaining sessions, all of which included experimental conditions with walking performance (i.e.; the second pretest session, the walking-practice sessions, and the two posttest sessions), took place in a different, large, well-lit room, with 1 or 2 research participants per session. The total size of this other room was $11 \times 12 \mathrm{~m}$. The final debriefing session took place in a conference room and included about 20-40 participants per session.

Recruitment and informed consent. Research participants were recruited through advertisements in local newspapers. When they came to the institute for the first time, individuals were given general information about the study and were told that participation in the study included the need to select a pair of comfortable shoes for the walking part of the experiment. Individuals were led to the walking tracks to provide them with a sense of the walking task, received an informed-consent form that contained all the relevant information, and were asked to carefully read through the form. Individuals willing to participate in the experiment signed the form and were reminded to bring an extra pair of comfortable shoes (e.g., walking shoes or jogging shoes; no high heels) to the next session (i.e., the first pretest session). Participants were given a receipt when they brought their shoes and were paid a fee of DM 25 for leaving their shoes at the institute for the duration of the experiment. Prior to the second pretest session, the pairs of shoes were prepared for walking track performance (see Footnote 2)

First pretest session. Participants were tested in groups of 3 to 5 individuals. They were given a computerized questionnaire to collect biographical background information. After that, the standard paper-andpencil version of Set II of the Raven Advanced Progressive Matrices test (Raven et al., 1983) and the shortened, modified form of the German

\footnotetext{
${ }^{2}$ Shoe soles were covered with sheets of graphite-coated foam (thickness: $4 \mathrm{~mm}$ ). In addition, the graphite-coated foam of the left shoe was again covered with aluminum tape, whereas the right shoe was covered with foam only. Both aluminum and graphite are electrically conductive materials, but graphite is much less conductive than aluminum. Therefore, resistance was higher when only the right foot was touching the ground. The foam had a very dense texture and did not interfere with normal walking behavior. Further details on the equipment used to assess parameters of walking performance can be obtained from Ulman Lindenberger.
} 
version of the Wechsler Vocabulary test (Lindenberger, Mayr, \& Kliegl, 1993; cf. Wechsler, 1982) were administered. Finally, baseline performance in serial recall was assessed with two 16-item word lists.

Second pretest session. This session served two purposes: (a) to obtain a baseline assessment of walking performance and (b) to assess possible differences in auditory comprehension (word identification) as a function of age group and experimental condition. Research participants were tested individually. First, research participants walked both tracks two times for $170 \mathrm{~s}$ under single-task conditions. They were instructed to "walk as quickly, safely, and accurately as possible, and to never stop walking until the end of the trial." In contrast to other parts of the experiment, the order of the tracks was fixed across participants; all participants first walked the oval track twice, and then the aperiodic track twice. After the baseline assessment of walking performance, an auditory comprehension task was administered to examine whether participants in all age groups were able to comprehend the auditory stimuli used in this study. Specifically, participants listened to a total of eight 16-word lists presented at a rate of $5 \mathrm{~s}$ per word over headphones using the infrared transmitter. Participants were instructed to immediately say back the word that they had just heard. Two lists were presented under each of the four experimental conditions: sitting, standing, walking the oval track, and walking the aperiodic track. There were four different orders of administration: (a) sitting, standing, oval track, aperiodic track; (b) oval track, sitting, aperiodic track, standing; (c) standing, aperiodic track, sitting, oval track; and (d) aperiodic track, oval track, standing, sitting. Within each age group, participants were equally and randomly distributed over the four different orders.

Instruction in memory performance with the MOL (Session 3). In Session 3, participants were introduced to the MOL (cf. Kliegl et al., 1989). First, participants were made familiar with the historical origins and the general procedure of the method. Then, they were given a handout that listed the 16 Berlin landmarks in serial order. Each of the landmarks was discussed in detail, and the resulting route was retraced on city maps. Participants were encouraged to create vivid images of the landmarks and were given examples of how to use mental imagery to establish a connection between the to-be-learned words and the landmarks. After that, participants practiced the technique with four word lists. To enhance acquisition of the mnemonic technique, participants were allowed to make use of the landmark list during encoding and retrieval. At the end of the session, participants were asked to rehearse the landmark list for the next session.

Memory training to criterion with the MOL. This block of sessions lasted between two and nine sessions, depending on individuals' rate of learning. At the beginning of each session, research participants were asked to recite the landmarks in correct serial order by heart. To encourage the use of the MOL, participants who were not yet able to correctly recite the landmarks were allowed to look at the landmark list during encoding and retrieval. Eight different word lists were given in each of the training sessions. Starting with the third practice session, training was terminated when research participants had attained the following criterion: They had worked without the landmark list in both the current and the previous sessions and they had attained a score of 12 (i.e., at least 12 out of 16 words at the correct serial positions) in at least one of the first four lists and a score of 12 in at least one of the second four lists. As a consequence, it was impossible to reach criterion prior to the second training session (i.e., the third session of MOL exposure). The maximum number of training sessions was fixed to nine. Individuals who did not reach the criterion in or before the ninth training session were excluded from the final sample (see the Results section for a description of sample attrition).

Walking practice. Half of the participants in each age group were asked to participate in four sessions of walking practice after completion of the pretest and memory training (see Table 1). Each of these sessions consisted of eight walking trials, four on the oval track and four on the aperiodic track. Each trial lasted for $170 \mathrm{~s}$. Track order within sessionsfour oval-track trials followed by four aperiodic-track trials and vice versa - was counterbalanced within and across individuals. Specifically, at each of the four sessions of walking practice, about half of the participants in each group started with the four oval-track trials, and the other half started with the four aperiodic-track trials. Moreover, for each participant, two sessions started with the four oval-track trials and the other two with the four aperiodic-track trials, the order of oval-track-first and aperiodictrack-first sessions being random.

As before, research participants were instructed to "walk as quickly, safely, and accurately as possible, and never to stop walking until the end of the trial." After each trial, research participants were given performance feedback regarding the speed and accuracy of their walking. Specifically, the following information was displayed on the computer screen: (a) distance covered in meters; given that participants walked for a constant amount of time $(170 \mathrm{~s})$, this is a measure of speed (i.e., number of meters $/ 170 \mathrm{~s}$ ); (b) the number of missteps to the right; and (c) the number of missteps to the left. Second, third, and fourth trials of a given track were added to the list as participants proceeded through the practice sessions. Thus, by the time participants had completed the fourth practice trial of a given track, the performance parameters of all four trials were listed on the screen, so that participants could easily see whether they had improved their performance in one or both parameters. The experimenter made sure that participants were paying attention to both speed and accuracy information when evaluating the feedback, and, if necessary, reminded participants to optimize both.

First posttest session. The purpose of this session was to assess walking and memorizing performance under single- and dual-task conditions following experimental manipulations (i.e., MOL training plus walking practice vs. MOL training plus no-intervention control). All research participants were exposed to a total of six different task conditions: encoding words with the MOL while (a) sitting, (b) standing, (c) walking the oval track, or (d) walking the aperiodic track (recall was always done under seated conditions); (e) oval track, walking only; (f) aperiodic track, walking only. For each of the six task conditions, two trials were administered in immediate succession. Under dual-task conditions, the first word was presented after $10 \mathrm{~s}$ of walking, and subsequent words were presented at the 10-s rate familiar from MOL training that is, $10 \mathrm{~s}+(16 \times 10 \mathrm{~s})=$ $170 \mathrm{~s}$. Under single-task walking conditions, participants walked for the same amount of time as under dual-task conditions (i.e., $170 \mathrm{~s}$ ).

To control for order effects, we grouped conditions into six blocks, and the six task blocks were combined to yield eight different administration orders. The six task blocks were as follows: (a) Block A: MOL with aperiodic track followed by MOL with oval track; (b) Block A': MOL with oval track followed by MOL with aperiodic track; (c) Block B: MOL sitting followed by MOL standing; (d) Block $B^{\prime}$ : MOL standing followed by MOL sitting; (e) Block C: oval track walking only followed by aperiodic track walking only; and (f) Block $C^{\prime}$ : aperiodic track walking only followed by oval track walking only. The eight different administration orders were: $\mathrm{ABC}, \mathrm{A}^{\prime} \mathrm{B}^{\prime} \mathrm{C}^{\prime}, \mathrm{BCA}, \mathrm{B}^{\prime} \mathrm{C}^{\prime} \mathrm{A}^{\prime}, \mathrm{ACB}, \mathrm{A}^{\prime} \mathrm{C}^{\prime} \mathrm{B}^{\prime}, \mathrm{CBA}, \mathrm{C}^{\prime} \mathrm{B}^{\prime} \mathrm{A}^{\prime}$. Research participants of each 3 (age group) $\times 2$ (walking practice) design cell were equally and randomly distributed over the eight different orders of administration. With respect to the group of old adults $(n=48)$, this procedure resulted in a perfectly stratified design with 3 research participants in each of the 2 (walking practice) $\times 8$ (posttest administration order) cells. With respect to the young $(n=45)$ and middle-aged $(n=47)$ groups, 2 or 3 of the resulting 16 design cells contained 2 , rather than 3 , individuals, respectively. ${ }^{3}$

${ }^{3}$ Both before and after the experimental part of the first posttest session, diastolic blood pressure, systolic blood pressure, and heart rate were measured. In analyses not reported here, we found that the cardiovascular measures taken before the experimental manipulations did not differ from those taken thereafter and did not interact with age group or any other factor. The relevant analyses can be obtained from Ulman Lindenberger. 
Table 3

Hearing Comprehension Performance as a Function of Measurement Occasion, Task Condition, and Age Group

\begin{tabular}{|c|c|c|c|c|c|c|c|c|}
\hline \multirow[b]{2}{*}{ Age group } & \multicolumn{4}{|c|}{ Pretest } & \multicolumn{4}{|c|}{ Posttest } \\
\hline & Seated & Standing & Oval track & Aperiodic track & Seated & Standing & Oval track & Aperiodic track \\
\hline \multicolumn{9}{|l|}{ Young } \\
\hline$M$ & 15.9 & 15.9 & 15.8 & 15.7 & 15.9 & 15.9 & 15.9 & 15.9 \\
\hline$\%$ without error & 83 & 83 & 61 & 61 & 83 & 78. & 87 & 83 \\
\hline \multicolumn{9}{|l|}{ Middle-aged } \\
\hline $\begin{array}{l}M \\
\text { of without error }\end{array}$ & $\begin{array}{l}15.8 \\
69\end{array}$ & $\begin{array}{l}15.9 \\
80\end{array}$ & $\begin{array}{l}15.7 \\
69\end{array}$ & $\begin{array}{l}15.8 \\
73\end{array}$ & $\begin{array}{l}15.8 \\
71\end{array}$ & $\begin{array}{l}15.9 \\
78\end{array}$ & $\begin{array}{l}16.0 \\
98\end{array}$ & $\begin{array}{l}15.9 \\
84\end{array}$ \\
\hline \multicolumn{9}{|l|}{ old } \\
\hline$M$ & 15.7 & 15.6 & 15.6 & 15.5 & 15.8 & 15.6 & 15.8 & 15.7 \\
\hline \% without error & 61 & 65 & 63 & 43 & 70 & 57 & 72 & 67 \\
\hline
\end{tabular}

Note. $\quad N=137$; data were missing for two young and one old participant. Maximum score $=16$. Scores are based on the average of two trials per condition. The first entry refers to the mean number of correctly identified words, the second entry to the percentage of research participants with a perfect score. Standard deviations for the mean number of correctly identified words are not reported because the data were highly skewed due to ceiling effects.

Second posttest session. The purpose of this session was to examine pretest-posttest changes in auditory comprehension and single-task walking performance. Content and procedure of this session were identical to the second pretest session.

\section{Results}

\section{Sample Attrition}

The original sample consisted of 177 participants. Of these, 140 took part in the entire experiment. Specifically, $5(10 \%)$ out of 52 young adults, $8(15 \%)$ out of 53 middle-aged adults, and $24(33 \%)$ out of 72 old adults did not complete the experiment. Age group differences in attrition rate were statistically reliable, $\chi^{2}(2, N=$ $177)=11.82, p<.01$. Reasons for dropout can be classified into four categories: (a) failure to reach the criterion for memory performance with the MOL within nine sessions of practice, young adults: $n=0$; middle-aged adults: $n=1$; old adults: $n=12$; (b) inability to walk on the tracks because of health problems (e.g., arthritis, high blood pressure, etc.), young adults: $n=0$; middleaged adults: $n=0$; old adults: $n=7$; (c) other forms of poor health, young adults: $n=0$; middle-aged adults: $n=2$; old adults: $n=4$; and (d) personal reasons (e.g., lack of interest, change of residence), young adults: $n=5$; middle-aged adults: $n=5$; old adults: $n=1$.

As revealed by this classification, dropout reasons differed considerably by age. Whereas all 5 of the young and 5 of the 8 middle-aged individuals discontinued the experiment for personal reasons, 12 of the 24 old adults had to discontinue the experiment because they were unable to attain criterion memory performance, and 7 because they were judged too frail to walk the experimental tracks. Compared with the 48 old adults who belonged to the final sample, the 12 old adults who did not reach criterion memory performance with the MOL were found to have lower scores on the Wechsler Vocabulary test, $F(1,58)=10.94, M S E=180.27, p<$ $.01, \eta^{2}=.16$, and marginally lower scores on the Raven Advanced Progressive Matrices test, $F(1,58)=5.07, M S E=97.54, p=$ $.028, \eta^{2}=.08 .^{4}$

We conclude that sample attrition among old adults in the present study was selective in the sense that it was related to the phenomena under investigation. Therefore, the walking and mem- ory performance of the sample of old adults who completed the entire experiment is likely to be positively biased. Given that we predict decrements in memory and walking performance as well as increments in DTCs with advancing age, and given that selective attrition was absent in the young and middle-aged adults groups, the positive selection bias among older adults works against our predictions by possibly reducing the magnitude of observable age differences with respect to the group of old adults.

\section{Word Comprehension Performance}

Table 3 displays mean word comprehension performance as a function of measurement occasion (pretest vs. posttest), age group, and encoding condition. The upper entries in the table represent the mean over the two trials administered under each condition. Values ranged from 15.5 (pretest, old adults, aperiodic track) to 16.0 (posttest, middle-aged adults, oval track). Given that the maximum attainable score was 16 , the observed mean values imply that close to all research participants were operating at or close to ceiling.

At the same time, further inspection of the means in Table 3 suggests that old adults performed slightly but consistently below the level of the middle-aged adults and young adults. To further examine this issue, we dichotomized auditory comprehension into perfect (1) versus less-than-perfect (0) levels of performance. Specifically, research participants who obtained a perfect score, that is, who recalled all 32 (i.e., $2 \times 16$ ) words of a given condition, were contrasted with participants who failed to identify at least one of the 32 words. The corresponding percentages for each experimental condition are shown in the second line of the age group panels in Table 3 .

The dichotomized data were analyzed with a repeated measures ANOVA with age group (young, middle-aged, or old) and walking practice (walking practice vs. no-intervention control) as betweensubjects factors and measurement occasion (pretest vs. posttest)

\footnotetext{
${ }^{4}$ All individuals who discontinued the experiment were individually debriefed. Also, individuals who did not reach criterion performance with the MOL completed a shortened version of the rest of the experiment to avoid negative emotional effects.
} 
and encoding condition (four encoding conditions) as withinsubjects factors. In addition to the two orthogonal contrasts for age group, three orthogonal contrasts were defined for experimental condition. The first contrast tested whether word comprehension under seated and standing encoding conditions differed from word comprehension under walking encoding conditions (aperiodic and oval track combined). The second contrast compared auditory word comprehension under seated versus standing encoding conditions, and the third auditory word comprehension under the oval-track versus aperiodic-track encoding conditions.

The second contrast for group was statistically reliable, $F(1$, $131)=16.09, M S E=0.26, p<.01, \eta^{2}=.11$, indicating that old adults' auditory word comprehension performance was below the level of auditory comprehension achieved by middle-aged adults. In addition, we observed a significant main effect of measurement occasion, $F(1,131)=12.43, M S E=0.20, p<.01, \eta^{2}=.09$, which was qualified by an interaction with the contrast of seated and standing versus oval-track and aperiodic-track encoding, $F(1$, $131)=15.66, M S E=0.18, p<.01, \eta^{2}=.11$. An inspection of Table 3 reveals that auditory word comprehension performance improved under walking but not under seated or standing encoding conditions. None of the remaining main effects or interactions were statistically reliable. ${ }^{5}$

In summary, research participants in all age groups displayed close-to-perfect or perfect auditory word comprehension performance. Despite this close-to-optimal level of word comprehension performance, there was some indication of improvement in word comprehension from pretest to posttest under walking encoding conditions. Finally, although old adults, on average, comprehended more than $97 \%$ of the words under all experimental conditions, their auditory word comprehension performance was slightly but reliably lower than the word comprehension performance of the other two groups.

Given the existence of these very small but reliable age differences in word comprehension, we routinely checked whether the pattern of age differences reported below was altered when auditory comprehension was entered as a covariate in the analyses. This was never the case.

\section{Training to Criterion in Serial Recall With the MOL}

Baseline performance prior to instruction and training in the MOL. On average, young adults, middle-aged adults, and old adults recalled $4.8(S D=2.7), 2.7(S D=1.6)$, and $1.9(S D=1.2)$ words per list in correct serial position, respectively. Data violated the variance homogeneity assumption (Cochran's $C=0.65, p<$ .01 ) and were transformed for further analysis by adding a constant of 0.1 and taking the natural logarithm. After transformation, the homogeneity assumption was met (Cochran's $C=0.39, p=.54$ ). Young adults performed above the level of middle-aged and old adults, $F(1,136)=40.10, M S E=0.23, p<.01, \eta^{2}=.23$. In contrast, the difference between middle-aged and old adults was not reliable, $F(1,136)=3.47, M S E=0.23, p>.05$.

Sessions to criterion. Young adults needed, on average, 4.2, middle-aged adults 5.5 , and old adults 6.8 sessions to reach criterion performance. These group differences were significant, young versus middle-aged and old adults, $F(1,137)=32.80$, $M S E=3.74, p<.01, \eta^{2}=.19$, and middle-aged versus old adults, $F(1,137)=10.17, M S E=3.74, p<.01, \eta^{2}=.07$. For at least two reasons, the observed group differences are likely to underestimate true age differences in learning to criterion. First, as a consequence of our procedure, all participants were trained for a minimum number of three sessions. Therefore, the observed distribution is likely to be left-censored in the sense that a substantial proportion of young adults may have been able to reach the criterion in the first or second session. Second, the group differences reported here do not take into account that 12 of the 72 old adults recruited for participation in this study were unable to attain criterion performance within 10 sessions of training (see the Sample Attrition section).

Age differences in MOL performance in the criterion session. Across the eight lists of the session in which the criterion was reached, young, middle-aged, and old adults recalled 13.0 $(S D=1.8), 11.5(S D=1.6)$, and $11.3(S D=1.5)$ words in correct serial position, respectively. The performance of young adults differed significantly from that of the other two groups, $F(1$, 137) $=30.90, M S E=2.68, p<.01, \eta^{2}=.18$; no significant differences were observed between the two older groups, $F(1$, 137) $=0.50, M S E=2.68, p=.48$. Thus, despite our attempts to eliminate age differences in episodic memory performance through criterion-references training, small but statistically reliable age differences subsisted after training to criterion. Differential exposure to the MOL was successful in the more limited sense that age-group differences in serial word recall were reduced compared with age-group differences at baseline, and that individuals in all groups performed at levels that were well beyond their baseline performance, suggesting that they did indeed adhere to the use of the mnemonic technique.

\section{Pretest-Posttest Comparisons of Walking Performance}

Walking speed. Table 4 displays walking speed (defined as the distance in meters covered in $170 \mathrm{~s}$ ) as a function of track, walking practice, measurement occasion, and age group. Our first analysis was restricted to pretest scores to examine whether walking speed at pretest differed as a function of walking-practice versus control conditions. In a 3 (age group) $\times 2$ (track) $\times 2$ (walking practice) ANOVA, the following reliable effects were found: (a) two main effects of group, young versus middle-aged and old, $F(1$, 133) $=25.07, M S E=2,139.64, p<.01, \eta^{2}=.16$, and middleaged versus old, $F(1,133)=12.84, M S E=2,139.64, p<.01$, $\eta^{2}=.09 ;$ (b) a main effect of track, $F(1,133)=303.24, M S E=$ $474.28, p<.01, \eta^{2}=.70$; and (c) an interaction of the middleaged versus old contrast with walking practice, $F(1,133)=9.47$, $M S E=2,139.64, p<.01, \eta^{2}=.07$. Follow-up analyses separately for each age group revealed that young adults in the walking-practice condition showed lower pretest performance than young adults in the control condition, $F(1,45)=7.79$, $M S E=1,638.06, p<.01, \eta^{2}=.15$. In contrast, old adults in the walking-practice condition showed higher pretest performance than old adults in the control condition, $F(1,45)=7.16$, $M S E=2,264.87, p=.010, \eta^{2}=.14$. Apparently, the assignment

\footnotetext{
5 The raw data of hearing comprehension performance (i.e., the mean number of correctly identified words per list) violated the assumption of variance-covariance homogeneity (Box's $M=412.64, p<.01$ ), but the dichotomized data did not (Box's $M=167.79, p<.50$ ). The two analyses yielded the same pattern of results.
} 
Table 4

Walking Speed as a Function of Measurement Occasion, Walking Practice, Track Type, and Age Group

\begin{tabular}{|c|c|c|c|c|c|c|c|c|}
\hline \multirow[b]{3}{*}{ Age group } & \multicolumn{4}{|c|}{ Oval track } & \multicolumn{4}{|c|}{ Aperiodic track } \\
\hline & \multicolumn{2}{|c|}{ Walking practice } & \multicolumn{2}{|c|}{ Control } & \multicolumn{2}{|c|}{ Walking practice } & \multicolumn{2}{|c|}{ Control } \\
\hline & Pretest & Posttest & Pretest & Posttest & Pretest & Posttest & Pretest & Posttest \\
\hline \multicolumn{9}{|l|}{ Young } \\
\hline$M$ & 183.8 & 254.4 & 216.8 & 225.9 & 142.1 & 197.5 & 155.8 & 172.4 \\
\hline$S D$ & 34.6 & 20.8 & 31.1 & 36.0 & 31.9 & 20.2 & 34.6 & 34.5 \\
\hline \multicolumn{9}{|l|}{ Middle-aged } \\
\hline$M$ & 171.0 & 220.2 & 185.2 & 213.3 & 128.1 & 157.8 & 145.4 & 161.1 \\
\hline$S D$ & 46.5 & 50.1 & 40.5 & 44.4 & 35.0 & 39.6 & 31.4 & 38.2 \\
\hline \multicolumn{9}{|l|}{ Old } \\
\hline$M$ & 170.4 & 184.0 & 139.5 & 163.5 & 121.7 & 132.6 & 100.2 & 111.8 \\
\hline$S D$ & 49.0 & 42.8 & 32.1 & 38.0 & 36.7 & 37.9 & 23.4 & 22.2 \\
\hline
\end{tabular}

Note. $\quad N=139$; data were missing for one old participant. Scores refer to the distance covered in $170 \mathrm{~s}$ and are based on the average of two trials per condition.

of research participants to walking-practice and control groups did not result in equivalent levels of pretest performance within age groups.

As can be seen in Table 4, age differences in walking speed were already quite large at pretest (e.g., averaged over walkingpractice conditions and tracks, young adults performed about one standard deviation above the mean of the old adults). To account for the magnitude of these age differences, research participants' gains in walking speed from pretest to posttest were expressed in a relative metric. Thus, performance gains for each of the two tracks were computed as follows:

$$
\text { Gain }=\left[\left(M_{\text {postrest }}-M_{\text {pretcst }}\right) / M_{\text {pretest }}\right] \times 100 \text {. }
$$

The 3 (age group) $\times 2$ (walking practice) $\times 2$ (track) data pattern violated homogeneity assumptions (Box's $M=112.41$, $p<.01$ ). After rank-transforming the gain scores (e.g., by assigning the highest rank to the person and condition with the largest relative gain in distance, etc.), data were more consistent with homogeneity assumptions (Box's $M=31.00, p>.01$ ). Two significant effects were found: (a) a main effect of walking practice, $F(1,133)=13.92, M S E=8,021.05, p<.01, \eta^{2}=.09$, and (b) an interaction of the first age-group contrast (i.e., young vs. middle-aged and old adults) with walking practice, $F(1$, 133) $=21.78, M S E=8,021.05, p<.01, \eta^{2}=.14$. An analysis based on the original relative gain scores, rather than ranked relative gains scores, yielded the same pattern of results, as did an analysis of covariance with posttest raw scores as dependent variables and pretest raw scores as covariates.

Figure 2 displays the critical walking practice by age group interaction for relative gain scores in the original metric. Gains in walking speed from pretest to posttest were largest for young adults in the walking-practice condition; participants in this group increased their walking speed by an average of $45 \%$. Within age groups, the difference between walking-practice and nointervention control conditions was very pronounced in young adults, $F(1,45)=76.64, M S E=1,879.00, \eta^{2}=.63, p<.01$. However, the same difference was not reliable for middle-aged adults, $F(1,43)=2.23, M S E=5,221.30, p>.10$, and old adults, $F(1,45)=1.22, M S E=4,985.08, p>.10$.

In sum, research participants in all age groups and walkingpractice conditions increased their walking speed from pretest to posttest. However, the four sessions of walking practice had a reliable effect on walking speed among young adults, but not among middle-aged and older adults.

Walking accuracy. Variables representing number of missteps were found to be very skewed, with skewness in the total sample ranging from 3.21 (oval track, pretest) to 4.78 (aperiodic track, pretest). For instance, with respect to the oval track at pretest, the

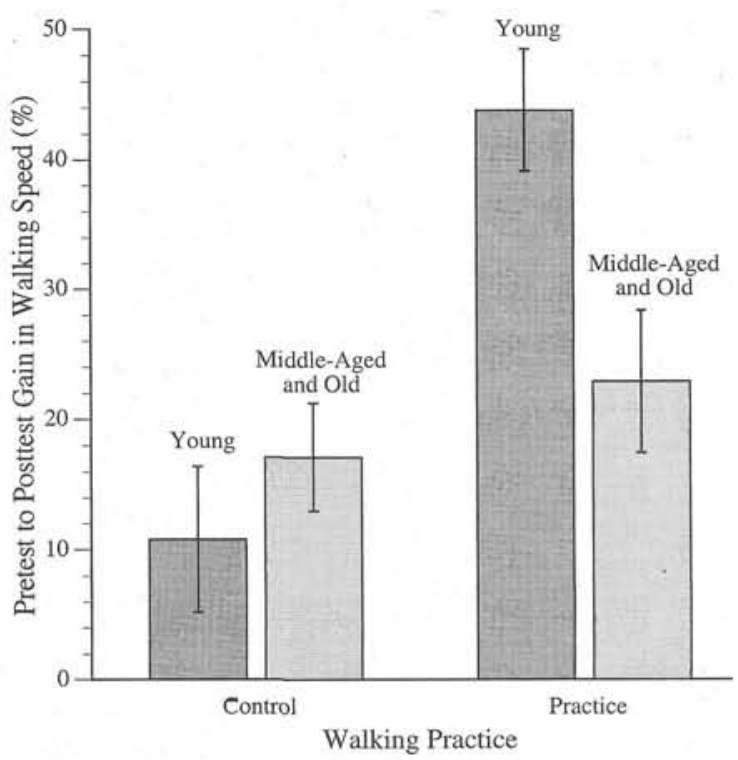

Figure 2. Gains in walking speed at posttest relative to pretest as a function of walking practice (practice vs. no-intervention control) and age group (young vs. middle-aged and old adults). Young adults in the walking-practice conditions showed larger gains in walking speed than all other groups. Error bars represent standard errors of the mean. 
Table 5

Walking Accuracy as a Function of Measurement Occasion, Walking Practice, Track Type, and Age Group

\begin{tabular}{|c|c|c|c|c|c|c|c|c|}
\hline \multirow[b]{3}{*}{ Age group } & \multicolumn{4}{|c|}{ Oval track } & \multicolumn{4}{|c|}{ Aperiodic track } \\
\hline & \multicolumn{2}{|c|}{ Walking practice } & \multicolumn{2}{|c|}{ Control } & \multicolumn{2}{|c|}{ Walking practice } & \multicolumn{2}{|c|}{ Control } \\
\hline & Pretest & Posttest & Pretest & Posttest & Pretest & Posttest & Pretest & Posttest \\
\hline \multicolumn{9}{|l|}{ Young } \\
\hline$M$ & 3.13 & 2.96 & 2.62 & 2.95 & 2.78 & 3.04 & 2.52 & 2.57 \\
\hline$S D$ & 0.82 & 0.71 & 0.92 & 0.87 & 0.74 & 0.56 & 0.68 & 0.75 \\
\hline \multicolumn{9}{|l|}{ Middle-aged } \\
\hline$M$ & 2.73 & 3.18 & 2.65 & 2.29 & 3.09 & 3.46 & 2.59 & 2.77 \\
\hline$S D$ & 1.01 & 0.41 & 0.70 & 0.85 & 0.54 & 0.82 & 0.62 & 0.66 \\
\hline \multicolumn{9}{|l|}{ Old } \\
\hline$M$ & 1.53 & 2.27 & 1.80 & 1.60 & 1.80 & 2.67 & 1.87 & 1.73 \\
\hline$S D$ & 0.52 & 0.88 & 0.86 & 0.99 & 0.78 & 0.90 & 0.74 & 0.88 \\
\hline
\end{tabular}

Note. Because of technical problems with the electronic device for misstep assessment at pretest, $N=102$. Scores represent the average of two trials per condition and are based on a 4-point scale derived from the average number of missteps per trial; $4=0$ missteps, $3=1-2$ missteps, $2=3-10$ missteps, $1=$ more than 10 missteps. Original misstep distributions were highly skewed.

following values were observed: mean number of missteps, young $=2.24$, middle-aged $=5.47$, old $=20.01$; median number of missteps, young $=0.88$, middle-aged $=0.88$, old $=8.88$; and maximum number of missteps, young $=14.25$, middleaged $=64.25$, old $=94.75$. Therefore, the original misstep variables were reduced to 4-point scales, with larger values representing higher levels of walking accuracy. Specifically, scales were defined as follows: 0 missteps $=4 ; 1-2$ missteps $=3 ; 3-10$ missteps $=2$; more than 10 missteps $=1$. Table 5 displays walking accuracy as captured by these 4-point scales as a function of track type, walking practice, measurement occasion, and age group.

In a repeated measures ANOVA with age group (three groups) and walking practice (two conditions) as between-subjects factors, measurement occasion (two occasions) and track (two tracks) as within-subject factors, and walking accuracy as the dependent variable, the following main effects were observed: Young adults versus old and middle-aged adults, $F(1,96)=14.29, M S E=1.38$, $p<.01, \eta^{2}=.13$; middle-aged adults versus old adults, $F(1$, 96) $=35.84, M S E=1.38, p<.01, \eta^{2}=.27$; walking practice, $F(1,96)=10.58, M S E=1.38, p<.01, \eta^{2}=.10$; measurement occasion, $F(1,96)=9.30, M S E=0.41, p<.01, \eta^{2}=.09$. These main effects were qualified by two interactions. First, the main effect of measurement occasion interacted with walking practice, $F(1,96)=9.30, M S E=0.41, p<.01, \eta^{2}=.11$, and was further qualified by a triple interaction with walking practice and age group (young vs. middle-aged and old adults), $F(1,96)=11.76$, $M S E=0.41, p<.01, \eta^{2}=.11$. As can be seen in Figure 3, this triple interaction was largely due to the fact that middle-aged and old adults in the walking-practice group showed significant improvements in walking accuracy, $F(1,25)=17.13, M S E=0.31$, $p<.01, \eta^{2}=.41$, whereas middle-aged and old adults in the control group as well as young adults in either of the two walkingpractice groups failed to show significant changes from pretest to posttest (all $p s>.10$ ).

In addition, the main effect of age was found to interact with track type. Contrary to expectations, age differences in walking accuracy were larger with the oval track than with the aperiodic track. Middle-aged and old adults were more likely to produce missteps on the oval track than on the aperiodic track, $F(1$, 57) $=13.16, M S E=0.12, p<.01, \eta^{2}=.19$. In contrast, differences in walking accuracy between the two tracks were not reliable for young adults $(p>.10){ }^{6}$

Summary of pretest-posttest comparisons of walking speed and walking accuracy. Averaged over the two tracks and both walking-practice conditions, young, middle-aged, and old adults walked $174(S D=31), 158(S D=36)$, and $133(S D=36) \mathrm{m}$ in $170 \mathrm{~s}$ at pretest, respectively. Without walking practice, the magnitude of these age differences was not significantly altered from pretest to posttest. With walking practice, however, age differences were magnified. Specifically, young adults in the walking-practice condition improved their walking speed by an average of $44 \%$, compared with $34 \%$ in middle-aged and $14 \%$ in old adults. With respect to walking accuracy, negative age differences were especially pronounced at pretest, for the oval track, and between middle-aged and old adults. In contrast to both middle-aged and old adults in the walking-practice condition, young adults did not show significant improvements in accuracy from pretest to posttest in either of the two walking-practice groups. Taken together, results indicate that young adults exhibited most of their practice gains in the speed parameter of walking performance, whereas

\footnotetext{
${ }^{6}$ Due to technical problems with the electronic device for misstep assessment at pretest, a large number of pretest observations were missing, reducing the effective number of individuals with information on all four variables in the analyses reported in this section from 140 to 102 . These technical problems did not interfere with the testing situation and were distributed about equally over age groups and experimental conditions. In control analyses in which missing data on the transformed variables were replaced by the corresponding cell means, the pattern of results was identical to the pattern reported in this section. We also wondered whether collapsing the misstep raw scores into a 4-point scale artificially reduced existing differences in walking accuracy between young and middle-aged adults. Additional analyses based on raw scores indicated that this was not the case.
} 


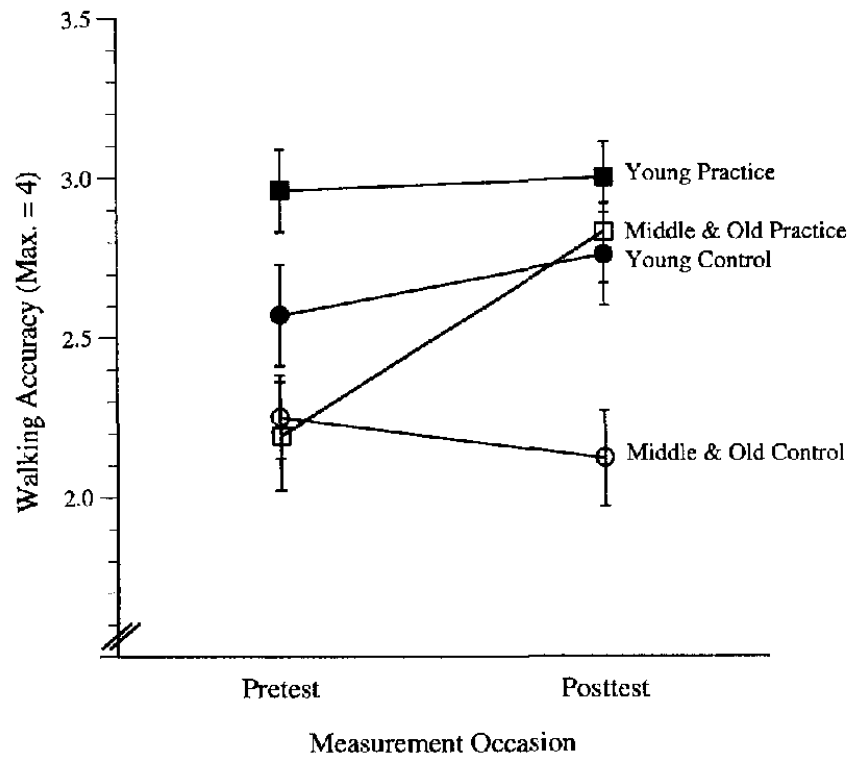

Figure 3. Pretest-posttest differences in walking accuracy as a function of walking practice (practice vs. no-intervention control) and age group (young vs. middle-aged and old adults). Middle-aged and old adults with walking practice improved their walking accuracy from pretest to posttest. In contrast, walking accuracy in both groups of young adults and in middle-aged and old adults without walking practice did not change from pretest to posttest. Walking accuracy reflects absence of missteps; $4=0$ missteps, $3=1-2$ missteps, $2=3-10$ missteps, $1=$ more than 10 missteps. Error bars represent standard errors of the mean. Max. = maximum.

middle-aged and old adults, who initially committed numerous missteps, improved relatively more in accuracy than in speed.

\section{DTCs}

Memory performance. Using a repeated measures ANOVA, the average number of words recalled in correct serial order was analyzed as a function of age group (three groups), walking practice (practice vs. control), and encoding condition (seated, standing, oval track, and aperiodic track). Three orthogonal contrasts were specified for encoding condition: (a) seated and standing versus oval and aperiodic track, (b) seated versus standing, and (c) oval versus aperiodic track. The main effects of age group were significant, young versus middle-aged and old adults, $F(1$, 134) $=53.71, M S E=24.32, p<.01, \eta^{2}=.29$, and middle-aged versus old adults, $F(1,134)=20.95, M S E=24.32, p<.01, \eta^{2}=$ .14. The contrast comparing seated and standing against oval- and aperiodic-track encoding conditions was also reliable, $F(1,134)=$ $136.55, M S E=6.62, p<.01, \eta^{2}=.50$, and was found to interact with the contrast testing young against middle-aged and old adults, $F(1,134)=13.02, M S E=6.62, p<.01, \eta^{2}=.09$. As can be seen in Table 6, recall performance in the context of walking encoding conditions was lower than recall performance in the context of seated and standing encoding conditions, and this difference was more pronounced among middle-aged and old than among young adults. Finally, recall was lower when individuals had to encode the to-be-learned words while walking on the aperiodic track than while walking on the oval track, $F(1,134)=46.18, M S E=5.15$, $p<.01, \eta^{2}=.26$. All other effects were not reliable $(p>.10)$.

A numerical interpretation of the critical age group by encoding condition interaction is difficult because age-group differences were already present under seated and standing encoding conditions (see Table 6). Therefore, we decided to further examine the nature of this interaction by expressing individuals' recall performance under walking encoding conditions relative to their performance under seated and standing encoding conditions. The latter two conditions did not differ from each other in any of the three groups (see Table 6). Hence, DTCs observed with the oval track were expressed as the percentage of loss in performance relative to performance under seated and standing recall conditions:

$$
\begin{aligned}
& \operatorname{DTCS}_{\mathrm{MOL}}=\left\{\left[\mathrm{NC}_{\text {mean(seated, standing) }}-\mathrm{NC}_{\text {oval }}\right] /\right. \\
& \left.\qquad \mathrm{NC}_{\text {mean(seated, standing) }}\right\} \times 100,
\end{aligned}
$$

where NC stands for the number of correctly recalled words. DTCs for the aperiodic track were computed accordingly:

$$
\begin{aligned}
& \operatorname{DTCs}_{\mathrm{MOL}}=\left\{\left[\mathrm{NC}_{\text {mean(seated, standing) }}-\mathrm{NC}_{\text {aperiodic }}\right] /\right. \\
& \left.\qquad \mathrm{NC}_{\text {mean(seated, standing) }}\right\} \times 100 .
\end{aligned}
$$

A 3 (age group) $\times 2$ (track) $\times 2$ (walking practice) repeated measures ANOVA revealed (a) that the aperiodic track was associated with larger DTCs than the oval track, $F(1,134)=35.63$, $M S E=492.07, p<.01, \eta^{2}=.21$; (b) that middle-aged and old adults showed greater DTCs than young adults, $F(1,134)=21.19$, $M S E=1,085.50, p<.01, \eta^{2}=.14$; and (c) that middle-aged and

\begin{tabular}{|c|c|c|c|c|c|c|c|c|}
\hline \multirow[b]{3}{*}{ Age group } & \multicolumn{8}{|c|}{ Encoding condition } \\
\hline & \multicolumn{2}{|c|}{ Sitting } & \multicolumn{2}{|c|}{ Standing } & \multicolumn{2}{|c|}{$\begin{array}{l}\text { Walking, } \\
\text { oval track }\end{array}$} & \multicolumn{2}{|c|}{$\begin{array}{l}\text { Walking, } \\
\text { aperiodic track }\end{array}$} \\
\hline & $M$ & $S D$ & $M$ & $S D$ & $M$ & $S D$ & $M$ & $S D$ \\
\hline Young & 13.0 & 2.9 & 13.3 & 2.4 & 12.9 & 2.8 & 10.5 & 3.6 \\
\hline Middle-aged & 11.7 & 3.2 & 12.1 & 3.0 & 9.7 & 3.3 & 7.9 & 3.6 \\
\hline Old & 9.5 & 3.0 & 9.6 & 3.2 & 7.2 & 3.8 & 5.8 & 3.2 \\
\hline
\end{tabular}

Table 6

Mean Number of Correctly Recalled Words as a Function of Track Type, Encoding Condition, and Age Group

Note. $N=140$. Maximum score $=16$. 
old adults did not differ significantly in DTCs, $F(1,134)=2.35$, $M S E=1,085.50, p=.13$. In agreement with the preceding analysis, performance did not vary reliably as a function of the two walking-practice conditions.

Figure 4 displays DTCs in memorizing as a function of age group and track. On average, middle-aged and old adults showed a $22 \%$ loss in serial recall with the less complex (oval) track and a $36 \%$ loss with the more complex (aperiodic) track. In contrast, young adults showed no loss at all with the less complex track $(0 \%)$ and a $19 \%$ loss with the more complex track.

Walking speed. Table 7 displays walking speed (i.e., number of meters $/ 170 \mathrm{~s}$ ) as a function of age group, walking practice, track, and task condition (single vs. dual). Given the magnitude of age-based differences in walking speed under single-task conditions, we refrained from an initial analysis of raw scores and restricted the analysis of DTCs to ratio scores. As before, these scores referred to the percentage of loss in walking speed under dual-task relative to single-task conditions. The 3 (age group) $\times 2$ (walking practice) $\times 2$ (track) data pattern violated homogeneity assumptions (Box's $M=40.79, p<.01$ ). Homogeneity assumptions were no longer violated with rank-transformed relative cost scores (e.g., the individual and condition with the highest relative cost score obtained the highest rank, etc.; Box's $M=14.11, p=$ .56). The only significant effect was a main effect of group, young adults versus middle-aged and old adults, $F(1,133)=16.09$, $M S E=9,037.60, p<.01, \eta^{2}=.11$. Figure 5 displays DTCs in walking speed averaged over both tracks as a function of age group. On average, middle-aged and old adults showed a $15 \%$ reduction in walking speed when having to encode the to-belearned words while walking. In contrast, young adults showed only a $8 \%$ reduction. ${ }^{7}$

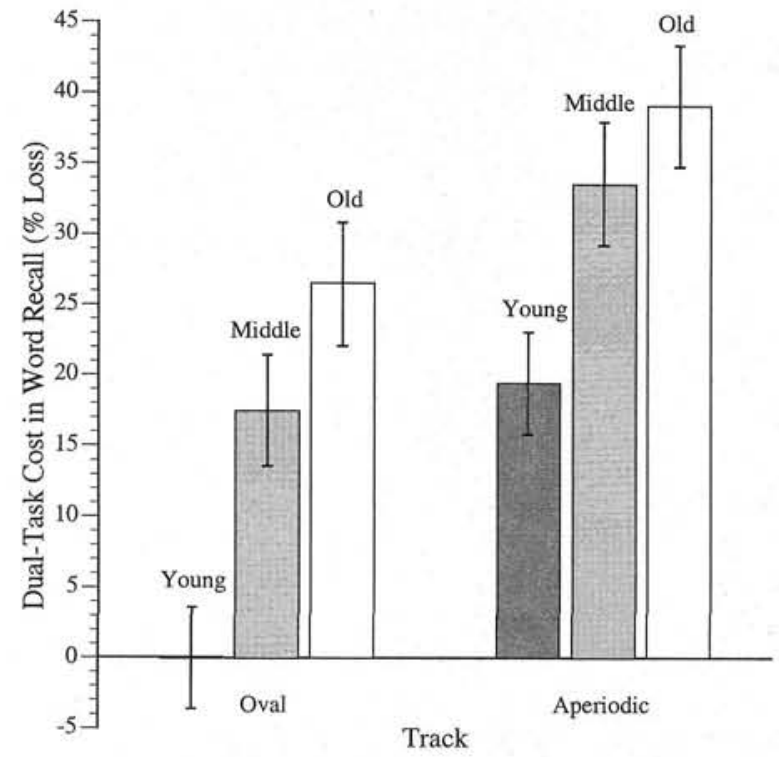

Figure 4. Dual-task costs in memory performance as a function of track and age group. Cost scores refer to the percentage of loss in serial word recall with the method of loci under walking encoding conditions (oval vs. aperiodic track) relative to the average of seated and standing encoding conditions. Middle-aged and old adults showed significantly higher costs than young aduits but did not differ from each other. Error bars represent standard errors of the mean.
Walking accuracy. Data were analyzed with a 3 (age group) $\times 2$ (walking practice) $\times 2$ (track) $\times 2$ (single- versus dual-task condition) repeated measures ANOVA, with the 4-point scale of walking accuracy serving as the dependent variable (see Table 8). Thus, in contrast to DTCs analyses regarding memory performance and walking speed, DTCs analyses of walking accuracy were performed with difference scores, rather than relative cost scores. This was done for two reasons. First, the use of a relative cost score is problematic with a 4-point scale. Second, our categorical recoding of missteps had effects similar to a log transformation; in fact, log-transformed missteps yielded the same pattern of results. Given that the original scale had been subjected to something akin to a $\log$ transformation, a subsequent ratio transformation of the resulting scales seemed inappropriate.

Both main effects of group were significant, young versus middle-aged and old adults, $F(1,131)=10.07, M S E=1.98, p<$ $.01, \eta^{2}=.07$, and middle-aged versus old adults, $F(1$, $131)=30.89, M S E=1.98, p<.01, \eta^{2}=.19$. Walking practice had a significant effect on walking accuracy, $F(1,131)=21.68$, $M S E=1.98, p<.01, \eta^{2}=.14$, indicating that participants with walking practice tended to be more accurate in their walking performance than participants in the control group. We also observed a significant main effect of track, $F(1,131)=8.20$, $M S E=0.30, p<.01, \eta^{2}=.06$, and an interaction between track and young versus middle-aged and old adults, $F(1,131)=7.50$, $M S E=0.30, p<.01, \eta^{2}=.05$. In agreement with results reported above, walking accuracy did not differ as a function of track in young adults, but middle-aged and old adults were more accurate on the aperiodic than on the oval track.

The main effect of single- versus dual-task condition was not reliable, $F(1,131)=1.76, M S E=0.24, p=.19$, but there was a significant interaction between this factor and the age group contrast comparing middle-aged and old adults, $F(1,131)=11.73$, $M S E=0.24, p<.01, \eta^{2}=.08$. As can be seen in Figure 6, young and middle-aged adults did not show significant DTCs in walking accuracy. In contrast, old adults were reliably less accurate under dual-task than under single-task conditions. Note that this is the only instance in which age-group differences in DTCs were observed between middle-aged and old participants, rather than between young participants and the two older groups.

Overall costs in dual-task performance. Baddeley et al. (1997) proposed the use of $z$-transformed scores to document and compare the magnitude of age differences in DTCs across task domains. Following this practice, the $z$-transformed accuracy and speed costs in the domain of walking were averaged and again $z$-transformed to reflect DTCs in walking. Corresponding $z$-transformed scores were computed for the memory domain. All $z$ scores were formed across tracks to preserve differences between tracks. Data were analyzed with a 3 (age group) $\times 2$ (track) $\times 2$ (domain: walking vs. memory) repeated measures ANOVA and are reported in Table 9. Note that the domain main effect has to be zero in this analysis because memorizing and walking are normed to the same metric. However, the domain factor can interact with

\footnotetext{
${ }^{7}$ The analysis based on relative cost scores without rank transformation, in which homogeneity assumptions were violated, produced the analogous result: young adults versus middle-aged and old adults, $F(1,133)=13.40$, $M S E=207.46, p<.01, \eta^{2}=.09$
} 
Table 7

Walking Speed as a Function of Track Type, Walking Practice, Task Condition (Single vs. Dual), and Age Group

\begin{tabular}{|c|c|c|c|c|c|c|c|c|}
\hline \multirow[b]{3}{*}{ Age group } & \multicolumn{4}{|c|}{ Oval track } & \multicolumn{4}{|c|}{ Aperiodic track } \\
\hline & \multicolumn{2}{|c|}{ Walking practice } & \multicolumn{2}{|c|}{ Control } & \multicolumn{2}{|c|}{ Walking practice } & \multicolumn{2}{|c|}{ Control } \\
\hline & Single & Dual & Single & Dual & Single & Dual & Single & Dual \\
\hline \multicolumn{9}{|l|}{ Young } \\
\hline$M$ & 254.4 & 234.2 & 225.9 & 208.7 & 197.5 & 179.9 & 172.4 & 158.6 \\
\hline$S D$ & 20.8 & 30.0 & 36.0 & 34.4 & 20.2 & 25.2 & 34.4 & 33.8 \\
\hline \multicolumn{9}{|l|}{ Middle-aged } \\
\hline$M$ & 220.2 & 184.0 & 213.3 & 183.2 & 157.8 & 138.2 & 161.1 & 130.8 \\
\hline$S D$ & 50.1 & 51.5 & 44.4 & 42.0 & 39.6 & 41.2 & 38.2 & 37.2 \\
\hline \multicolumn{9}{|l|}{ Old } \\
\hline$M$ & 184.3 & 164.3 & 161.7 & 134.0 & 132.9 & 117.9 & 112.1 & 93.2 \\
\hline$S D$ & 43.7 & 47.5 & 38.3 & 38.4 & 38.7 & 38.2 & 21.7 & 24.6 \\
\hline
\end{tabular}

Note. $\quad N=139$; data were missing for one old participant. Scores refer to walking distance (in meters) in $170 \mathrm{~s}$. Under dual-task conditions, participants had to encode a list of 16 to-be-learned words presented at a rate of $10 \mathrm{~s}$ per word while walking.

any of the remaining factors. DTCs were larger in middle-aged and old adults than in young adults, $F(1,136)=40.38, M S E=1.19$, $p<.01, \eta^{2}=.23$; the difference between middle-aged and old adults was not significant at the .01 level, $F(1,136)=5.01$, $M S E=1.19, p=.027, \eta^{2}=.04$. DTCs with the aperiodic track were larger than with the oval track, $F(1,136)=19.39$, $M S E=0.59, p<.01, \eta^{2}=.12$, and this main effect was qualified by a track by domain interaction, $F(1,136)=14.19, M S E=0.51$, $p<.01, \eta^{2}=.09$. Interactions of age group with track or domain were not reliable $(p>.40)$. In line with the earlier results, post hoc analyses within domains revealed that the track effect was reliable for memorizing, $F(1,136)=34.44, M S E=0.52, p<.01$, $\eta^{2}=.21$, but not for walking, $F(1,136)=0.42, M S E=0.58$, $p>.50$.

Overall, the effect size of the age-associated increase in DTCs averaged over the two tracks, expressed as the difference to young adults over the standard deviation of young adults, was 0.98 standard deviation units for middle-aged adults and 1.47 standard deviations for old adults.

Intercorrelations among DTCs. Table 10 displays correlations among DTCs in memorizing and walking speed; DTCs in walking accuracy were not included in the table because they did not differ from zero in young and middle-aged adults. DTCs were positively correlated within domains but unrelated across domains. The moderately high correlations within task domains suggest that DTCs were assessed in a sufficiently reliable manner. The absence of significant correlations between DTCs across domains may come as a surprise. Note, however, that the sign and magnitude of such interindividual across-domain DTCs correlations is a function of at least two opposing influences: individual differences in relative emphasis on the two tasks, which would induce a negative correlation, on the one hand, and individual differences in "attentional resources," which would induce a positive correlation, on the other. Apparently, these diverging influences canceled each other out in the present study.

\section{Microanalyses of Dual-Task Dynamics: Evidence for Periodic Attentional Shifts Between Memorizing and Walking}

The last set of analyses concerns step frequency as a function of time after onset of to-be-learned words. Step frequency was chosen because it showed a strong relationship to walking speed $(r=.80)$ and could be reliably assessed within relatively short time intervals.

Throughout this study, the 16 to-be-learned words of a given list were presented at a constant rate of $10 \mathrm{~s}$. That is, when participants

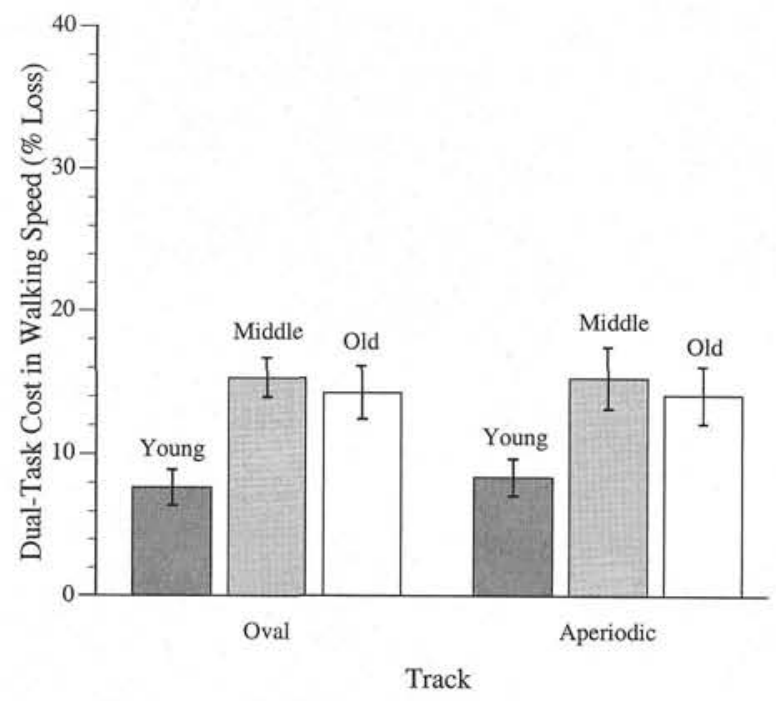

Figure 5. Dual-task costs in walking speed as a function of track and age group. Cost scores refer to the percentage of loss in walking speed under dual-relative to single-task conditions. Middle-aged and old adults showed significantly higher costs than young adults but did not differ from each other. Under dual-task conditions, participants had to encode a 16-item word list. Error bars represent standard errors of the mean. 
Table 8

Walking Accuracy as a Function of Track Type, Walking Practice, Task Condition (Single vs. Dual), and Age Group

\begin{tabular}{|c|c|c|c|c|c|c|c|c|}
\hline \multirow[b]{3}{*}{ Age group } & \multicolumn{4}{|c|}{ Oval track } & \multicolumn{4}{|c|}{ Aperiodic track } \\
\hline & \multicolumn{2}{|c|}{ Walking practice } & \multicolumn{2}{|c|}{ Control } & \multicolumn{2}{|c|}{ Walking practice } & \multicolumn{2}{|c|}{ Control } \\
\hline & Single & Dual & Single & Dual & Single & Dual & Single & Dual \\
\hline \multicolumn{9}{|l|}{ Young } \\
\hline$M$ & 2.88 & 3.12 & 3.00 & 2.71 & 3.00 & 3.24 & 2.62 & 2.67 \\
\hline$S D$ & 0.73 & 0.78 & 0.84 & 0.72 & 0.58 & 0.72 & 0.74 & 0.73 \\
\hline \multicolumn{9}{|c|}{ Middle-aged } \\
\hline$M$ & 3.05 & 3.10 & 2.42 & 2.71 & 3.30 & 3.25 & 2.75 & 2.71 \\
\hline$S D$ & 0.76 & 0.85 & 1.02 & 0.91 & 0.73 & 0.79 & 0.79 & 0.86 \\
\hline \multicolumn{9}{|l|}{ Old } \\
\hline$M$ & 2.48 & 2.17 & 1.67 & 1.50 & 2.87 & 2.52 & 1.92 & 1.58 \\
\hline$S D$ & 0.99 & 0.94 & 0.87 & 0.78 & 0.97 & 0.95 & 0.83 & 0.78 \\
\hline
\end{tabular}

Note. $\quad N=137$; data for one young, one middle-aged, and one old participant were missing. Scores represent the average of two trials per condition and are based on a 4-point scale derived from the average number of missteps per trial; $4=0$ missteps, $3=1-2$ missteps, $2=3-10$ missteps, $1=$ more than 10 missteps. Original misstep distributions were highly skewed. Under dual-task conditions, participants had to encode a list of 16 to-be-learned words presented at a rate of $10 \mathrm{~s}$ per word while walking.

heard a given word, they had about $10 \mathrm{~s}$ to access the corresponding landmark location, form a mental association between the landmark and the to-be-learned word (most likely a compound mental "image"), and prepare processing of the next word. On the basis of past research (Baddeley \& Lieberman, 1980; Lindenberger et al., 1992), we assumed that the formation of the landmark-noun association was the most demanding component in this processing sequence. Therefore, we expected that participants would periodically shift their attention to the memory task after the onset of the 10 -s interval in order to form a memorable mental image, provoking a decrease in step frequency. To examine this issue, we aggregated the 3210 -s encoding intervals per condition (i.e., two trials with 16 to-be-learned words each) and divided them into four segments of $2.5 \mathrm{~s}$ each. Our expectation was that walking step frequency would be lower at later intervals. In addition, we also examined whether step frequency varied as a function of recall status of to-be-learned words (i.e., recalled vs. not recalled).

An ANOVA with age group (three groups) and walking practice (two conditions) as between-subjects factors; track (two tracks), temporal segment (four segments), and recall performance (recalled vs. not recalled) as within-subject factors; and number of steps as the dependent variable was used to analyze the data. The data pattern violated homogeneity assumptions (Box's $M=$ $506.70, p<.01$ ). After we rounded step frequency values to the next full step and recoded values lower than 2 to 2 and values higher than 5 to 5 , data corresponded more closely to homogeneity assumptions (Box's $M=396.29, p>.01$ ). Three main effects were observed: (a) a main effect of group, indicating that middleaged and old adults made fewer steps than young adults, $F=(1$, $131)=15.75, M S E=4.70, p<.01, \eta^{2}=.11$; (b) a main effect of track, indicating that step frequency was lower when participants were walking on the aperiodic than when they were walking on the oval track, $F=(1,131)=239.03, M S E=0.53, p<.01$, $\eta^{2}=.65$; and (c) a main effect of temporal segment, $F=(3$, $393)=350.49, M S E=0.22, p<.01, \eta^{2}=.73$. The only other statistically reliable effect was an interaction between track and temporal segment, $F=(3,393)=5.24, M S E=0.17, p<.01$, $\eta^{2}=.04$. All remaining effects were statistically not reliable (i.e., all $p s>.06$ ). When the analysis was performed on untransformed data (which violated homogeneity assumptions), the three main effects were also statistically reliable, but the track by temporal segment interaction was not. For this reason, we refrain from an interpretation of this interaction.

Figure 7 displays step frequency raw scores as a function of age group and temporal segment. The results are consistent with the

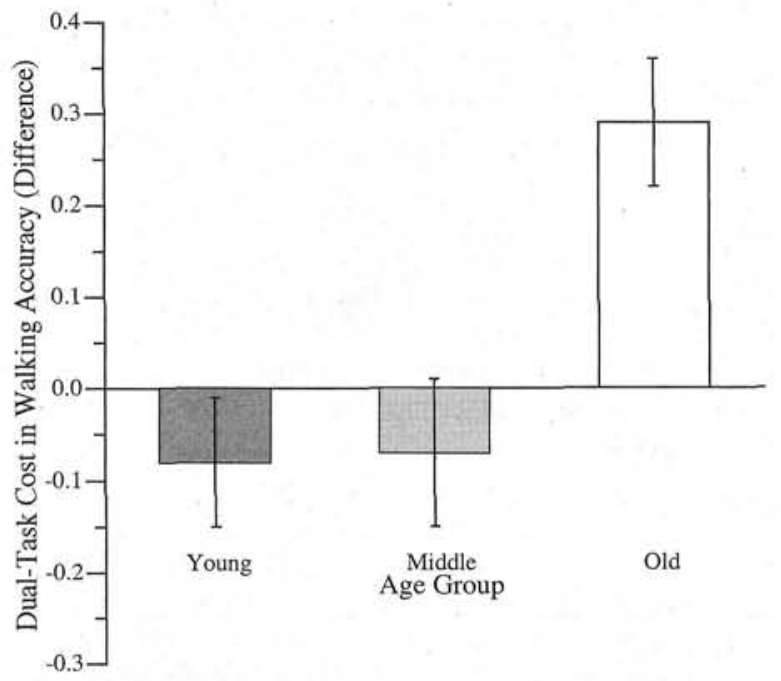

Figure 6. Dual-task costs in walking accuracy as a function of age group. Cost scores represent the difference in walking accuracy between singleand dual-task conditions. Old adults showed significantly higher costs than middle-aged adults. Under dual-task conditions, participants had to encode a 16-item word list. Walking accuracy reflects absence of missteps; $4=0$ missteps, $3=1-2$ missteps, $2=3-10$ missteps, $1=$ more than 10 missteps. Error bars represent standard errors of the mean. 
Table 9

Normalized Dual-Task Costs as a Function of Track, Domain, and Age Group

\begin{tabular}{|c|c|c|c|c|c|c|c|c|c|c|}
\hline \multirow[b]{3}{*}{ Age group } & \multicolumn{4}{|c|}{ Oval track } & \multicolumn{4}{|c|}{ Aperiodic track } & & \\
\hline & \multicolumn{2}{|c|}{ Memorizing } & \multicolumn{2}{|c|}{ Walking } & \multicolumn{2}{|c|}{ Memorizing } & \multicolumn{2}{|c|}{ Walking } & \multicolumn{2}{|c|}{ Combined } \\
\hline & Cost & $S D$ & Cost & $S D$ & Cost & $S D$ & Cost & $S D$ & Cost & $S D$ \\
\hline Young & -0.74 & 0.81 & -0.35 & 0.76 & -0.11 & 0.83 & -0.47 & 0.80 & -0.42 & 0.51 \\
\hline Middle-aged & -0.17 & 0.88 & -0.04 & 1.19 & 0.35 & 1.00 & 0.16 & 1.07 & 0.08 & 0.61 \\
\hline Old & 0.12 & 1.00 & 0.30 & 0.93 & 0.50 & 0.96 & 0.40 & 0.92 & 0.33 & 0.52 \\
\hline
\end{tabular}

Note. $\quad N=139$. Scores represent normed costs across walking and memorizing. Specifically, the $z$-transformed accuracy and speed costs in the domain of walking were averaged, and the resulting scores were again $z$-transformed. Corresponding cost scores were computed for the memory domain. Transformations were computed across tracks to preserve differences in costs between tracks. To arrive at an overall cost score (right column), we averaged scores across tracks and domains.

main prediction. On both tracks, participants lowered their step frequency over the four consecutive 2.5 -s segments: linear trend, $F=(1$, $131)=1,071.43, M S E=0.19, p<.01, \eta^{2}=.89$. Perhaps surprisingly, the magnitude of this reduction did not depend on the recall status of the words. When expressing step frequency in the fourth segment as a proportion of step frequency in the first segment, research participants in all age groups reduced their frequency by about $23 \%$. Apparently, then, there was a systematic shift in relative task emphasis over the 10-s interval (cf. Maylor et al., in press).

\section{Discussion}

The primary prediction of this study was that DTCs increase from early adulthood to old age when a relatively difficult locomotion task and an episodic memory task are performed in conjunction, rather than in isolation. Specifically, individuals were asked to walk on narrow tracks of differing path complexity while encoding word lists with the use of a mnemonic technique. The results of the present study demonstrate that this particular form of dual-task performance increases in difficulty across the adult life span.

\section{Summary of Major Findings}

Age differences in DTCs. With advancing age, participants showed greater reductions in memory accuracy when they were walking (see Figure 4), rather than sitting or standing, during mnemonic encoding. In addition, they showed greater reductions in speed of walking (see Figure 5) and accuracy of

Table 10

Intercorrelations Among Dual-Task Costs

\begin{tabular}{lcccc}
\hline \multicolumn{1}{c}{ Variable } & 1 & 2 & 3 & 4 \\
\hline 1. Walking speed, oval track & - & $.49^{*}$ & .17 & .12 \\
2. Walking speed, aperiodic track & $.46^{*}$ & $-\overline{11}$ & -.03 & .04 \\
3. Memorizing, oval track & .07 & -.11 & $\overline{-}$ & $.44^{*}$ \\
4. Memorizing, aperiodic track & .05 & -.02 & $.38^{*}$ & - \\
\hline
\end{tabular}

Note. First-order correlations are shown above the main diagonal; correlations controlled for age group are shown below the main diagonal.

$* p<.01$. walking (see Figure 6) when they had to simultaneously engage in memonic encoding operations. With respect to memorizing and walking speed, these age-based increments in DTCs were already present in 40 - to 50 -year-old adults. With respect to walking accuracy, they were confined to 60- to 70-year-old participants. As expected, walking on a more complex track
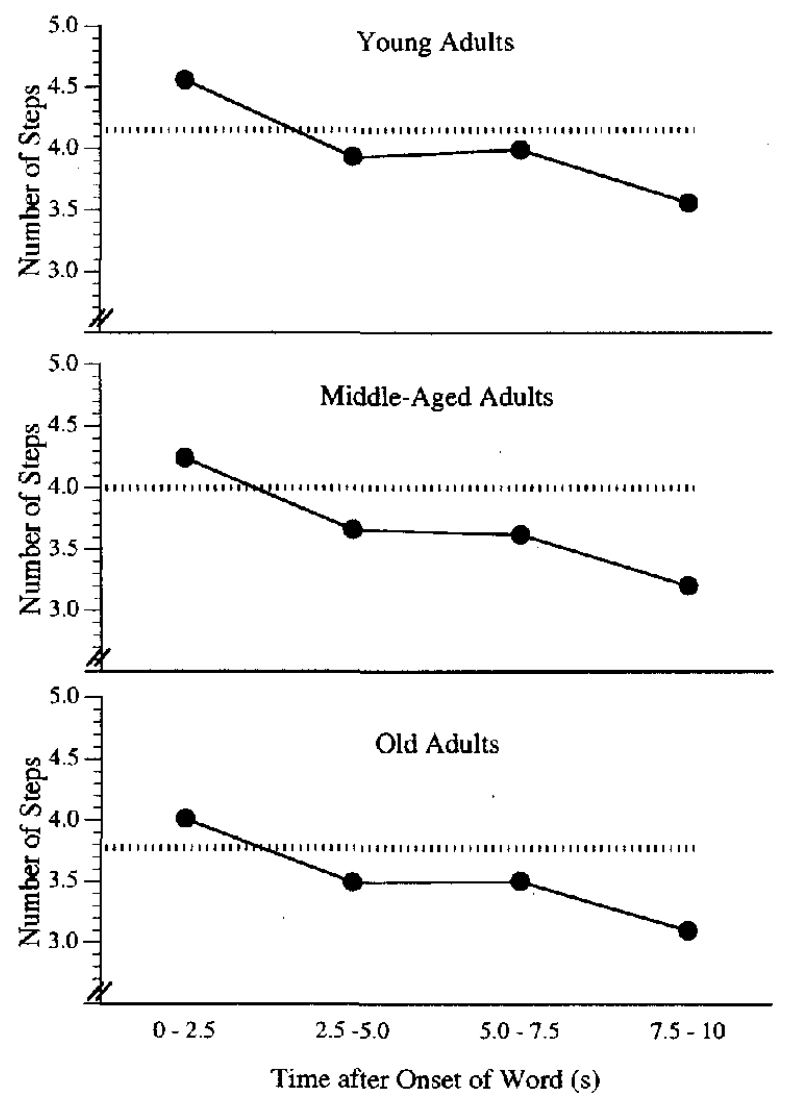

Figure 7. Step frequency under dual-task conditions as a function of age group and time after onset of the to-be-learned word. Step frequency decreased toward the end of the 10-s encoding interval. This tendency did not interact with age group. For purpose of comparison, dashed horizontal lines represent step frequency under single-task (i.e., walking-only) conditions. 
induced greater DTCs in memorizing than walking on a less complex track. In fact, for young adults walking on the less complex track, DTCs in memory performance were completely absent (see Figure 4). ${ }^{8}$

Absence of interactions with walking practice. Walking practice led to increments in both walking speed and walking accuracy in all three age groups. In contrast to middle-aged and old adults, young adults were already operating at close-to-perfect levels of walking accuracy at pretest and showed larger gains than middleaged and old adults in walking speed but smaller gains (in fact, no gains) in walking accuracy. Somewhat unexpectedly, walking practice failed to reduce DTCs in general, and age differences in DTCs in particular. In other words, DTCs in individuals with prior walking practice did not differ reliably from DTCs in individuals without such practice, and the same was true for age differences in DTCs. Thus, DTCs and age differences therein, were neither removed nor reduced by moderate amounts of self-guided practice in the relevant sensorimotor skill. We do not know, however, whether continued exposure to the dual-task situation itself would have produced a different outcome (cf. Kramer et al., 1995; Kray \& Lindenberger, 2000).

\section{Limitations and Qualifications of the Present Findings}

Continued existence of age differences in memory performance after training to criterion. Unfortunately, the provision of differential amounts of training with the MOL did not eliminate agegroup differences in memory performance. First, age groups differed in the average number of correctly recalled words in the session in which the criterion was attained. Technically, this was possible because the criterion was not defined as a perfect (i.e., flawless) level of performance. Almost certainly, a higher criterion level would have further increased the percentage of older adults who failed to reach the criterion, thereby aggravating the differential selection problem. Thus, the rather lenient criterion set in this study was a compromise between the goal of attaining perfect performance equality across age groups, on the one hand, and the goal of not losing too many old adults, on the other.

In addition, old adults, but not middle-aged and young adults, evinced a decrement in average performance from the criterion session to seated conditions at posttest: old, 11.3 vs. 9.5 , $t(47)=4.16, p<.01$; middle-aged, 11.5 vs. $11.7, t(44)=-0.41$, $p>.50$, young, 13.0 vs. $13.0, t(46)=0.10, p>.50$. Older adults inability to maintain criterion levels of performance at posttest could have several causes. First, the younger age groups reached criterion performance at an earlier phase of the skill-acquisition process and may have continued to improve on the task after having reached the criterion. Second, memory training and posttest took place in different rooms, and the skilled memory performance of older participants may have suffered more from this contextual variation than the memory performance of younger participants (cf. Winocur \& Moscovitch, 1983). Third, one may wonder whether skill (i.e., memory strategy) maintenance over time differed as a function of age group. Given that the posttest took place about 2 weeks after participants had reached criterion memory performance, it is conceivable that some of the older adults were no longer able to use the MOL. However, this explanation is unlikely for two reasons. First, it has been shown repeatedly that older adults are able to maintain the use of a mnemonic skill such as the MOL over several weeks, months, and even years (Baltes \& Kliegl, 1992; Stigsdotter Neely \& Bäckman, 1993). Furthermore, at the beginning of the posttest session, research participants were asked to quickly recite the list of location cues by heart. All research participants were able to recite the list without error, which indicates that the sequence of landmark cues was well overlearned by all participants.

The observed age differences in DTCs would be more easily interpretable if the training-to-criterion procedure had fully eliminated any age-group differences in memory performance under standard encoding conditions. One way to examine the possible influence of age-group differences in single-task performance on age-group differences in DTCs is to perform post hoc analyses with individuals of different ages who display similar levels of single-task performance. Therefore, we selected individuals who, on average, recalled no fewer than 9 and no more than 13 items in correct serial position under single-task (i.e., seated or standing) encoding conditions. This left us with 18 young, 18 middle-aged, and 20 old adults with an average correct recall of, respectively, $11.4(S D=1.1), 10.9(S D=1.3)$, and $11.2(S D=1.2)$ words under single-task conditions. In line with our predictions, DTCs in memory performance continued to be more pronounced in middle-aged and old than in young adults, $F=(1,53)=7.48$, $M S E=1,265.08, p<.01, \eta^{2}=.12$, and the overall pattern of findings, including DTCs in walking speed and walking accuracy, matched the pattern observed with the full data set. In the light of this additional analysis, it seems unlikely that the pattern of age differences observed in the present study would have been markedly different if age differences in memorizing under single-task conditions had been fully eliminated through differential exposure to the MOL.

Equal-emphasis instructions. In the critical dual-task conditions at posttest, participants were instructed to give equal importance, in terms of performance outcomes, to walking and memorizing (e.g., "to walk as quickly and accurately as possible, and to remember as many words as possible"). Thus, participants were asked to optimize performance in both tasks. This instruction does not directly correspond to typical dual-task instructions in which participants are asked to invest an equal proportion of cognitive effort into both tasks (cf. Salthouse, Rogan, \& Prill, 1984). Specifically, the two types of instructions would be identical only if the functions relating cognitive effort to performance were identical across tasks (cf. Norman \& Bobrow, 1975).

We lack the critical information to judge whether age-based increases in cognitive demand characteristics, under the specific experimental conditions of this experiment, were more pronounced for walking or memorizing. Age-comparative effort-performance functions of walking and memorizing (cf. Kliegl, Mayr, \& Krampe, 1994; Norman \& Bobrow, 1975; Salthouse et al., 1984)

\footnotetext{
${ }^{8}$ Statements regarding direct comparisons between young and middleaged adults were backed up by post hoc analyses restricted to these two age groups. With respect to the two analyses concerning walking accuracy (i.e., training gains and DTC), age-group differences were present for young versus middle-aged and old adults but absent for young versus middle-aged adults. For all other analyses, age differences that were statistically reliable between young versus middle-aged and old adults were also reliable between young and middle-aged adults.
} 
would be needed to provide strong data on this issue. However, subjective reports are also useful, especially if elicited during or immediately after the relevant activity (cf. Ericsson \& Simon, 1993). Therefore, at the end of the posttest session, we asked participants to indicate what percentage of their total attention they had invested into walking versus memorizing under dual-task conditions. An ANOVA with age group (three groups) and walking practice (two conditions) as independent variables and emphasis on memorizing as the dependent variable yielded no significant effects (all $p s>.35$ ). Averaged over age groups and walkingpractice conditions, participants said that they had invested $64 \%$ of their attention in memorizing (95\% confidence interval: $61.4 \%$ to $66.8 \%$ ). This is consistent with the assumptions (a) that memorizing was cognitively more demanding than walking; (b) that an equal-emphasis instruction phrased in terms of performance outcomes did not directly correspond to the typical dual-task instruction to "equally divide" one's attention; and (c) that the difficulty of the two tasks increased at about the same rate with advancing age. Experimental manipulations of relative task emphasis are needed to validate these assumptions.

Speed-accuracy trade-offs in walking performance. In this study, age differences in accuracy of walking performance were substantial both with and without simultaneous encoding activity with the MOL. Specifically, participants in the oldest age group were much more likely to step outside the boundaries of the two tracks than middle-aged and young adults (see Tables 5 and 8). This age-associated decrease in the accuracy of walking performance is probably due to age-based decrements in the ability to maintain balance during locomotion (Shumway-Cook et al., 1997). As a consequence, it seems likely that older adults had to limit their speed of walking under all experimental conditions to a greater extent than young and middle-aged adults to remain on track. This observation may help to explain why middle-aged adults had greater DTCs in the speed component of walking than young adults, whereas old adults had greater DTCs in the accuracy component of walking performance than middle-aged adults (see Figures 5 and 6). Overall, however, DTCs increased with age in both components of walking behavior. Therefore, speed-accuracy trade-offs do not offer a viable explanation for the existence of age differences in DTCs.

Localization of observed age differences in DTCs. In this study, age differences in DTCs were predicted for two reasons: (a) because locomotion requires an increasing amount of cognitive control and supervision with advancing age and (b) because locomotion is increasingly dependent on attention-demanding visuospatial processing. According to these predictions, the observed age differences in DTCs could primarily reflect processing conflicts at the level of more or less supramodal processes of task supervision and maintenance (e.g., "executive" mechanisms; cf. Shallice \& Burgess, 1993), or conflicts at the level of attentiondemanding processes in the visuospatial modality, or both. More detailed age-comparative investigations of locomotion and memory are needed to discriminate among these alternatives.

\section{The Permeation of Behavior With Cognition: A General Process of Normal Aging?}

Despite large differences in tasks and procedures, the results of the present study are in good agreement with the results of earlier age- comparative dual-task studies on posture control, locomotion, and cognition that were reviewed in the Introduction. In addition, they are in line with more clinically oriented observations on interindividual differences in locomotion among older individuals. For instance, in a study on the incidence rates of falls among elderly nursing-home residents $(N=58$, mean age $=80.1$ years, $S D=6.1)$, LundinOlsson, Nyberg, and Gustafson (1997) found that 10 out of 12 of the individuals who stopped walking when starting a conversation fell at least once during a 6-month follow-up period, whereas 35 out of 46 of those who did not stop walking also did not experience a fall within that period; this association between stopping while talking and falls was statistically reliable. In a further study $(N=42$, mean age $=79.7$ years, $S D=6.1$ years), Lundin-Olsson, Nyberg, and Gustafson (1998) asked participants to rise from an armchair, walk $3 \mathrm{~m}$, turn around, and sit down again, both with and without an added manual task, which was to carry a glass of water while walking. The time difference between single- and dual-task conditions was associated with greater dependency in activities of daily living, decreased postural control in standing, lower scores on several cognitive tests, and an increased risk of falling during a 6-month follow-up period.

In light of this remarkable consistency across studies, we propose that age-associated increments in sensorimotorcognitive DTCs point to a more general phenomenon in adult development and aging: the age-associated permeation of behavior with cognition. According to this view, which is reminiscent of Alan Welford's early observations (e.g., Welford, 1958, pp. 186-187), sensory and motor aspects of performance are increasingly in need of cognitive control and supervision because of frailty, sensory losses, and lower level problems in sensorimotor integration and coordination. ${ }^{9}$

At the same time, mechanisms related to cognitive supervision and control also decrease in efficiency with advancing age, and some, but not all of them appear to be disproportionately compromised in old age (e.g., Kramer, Hahn, \& Gopher, 1999). Hence, aging individuals are confronted with a quandary in the sense that cognitive control mechanisms are more and more called for but less and less able to counteract the wide-ranging adverse consequences of sensory and motor deficits. In addition to other factors, such as the possible existence of a common set of brain-related causes operating across sensory, motor, and cognitive domains (Baltes \& Lindenberger, 1997; Lindenberger \& Baltes, 1994), this increasing dependency of sensory and motor performance on cognitive control would also help to explain the strong correlational link between sensory, motor, and intellectual performance in old age (Anstey et al., 1997; Anstey et al., 1993; Baltes \& Lindenberger, 1997; Lindenberger \& Baltes, 1994, 1997 ) and would contribute to an aging-induced contraction of "functional cerebral space" (Kinsbourne \& Hicks, 1978; Korteling, 1993). Future work should examine the developmental dynamics of this quandary and study the extent to which compensatory strategies, including the skilled use of external aids, may attenuate its maladaptive consequences ( $\mathrm{Li}$, Lindenberger, Freund, \& Baltes, 2000; cf. Baltes, 1997).

\footnotetext{
${ }^{9}$ In comparison to the "resource allocation" metaphor, the permeation metaphor puts greater emphasis on structural changes in the processing composition of sensorimotor tasks (e.g., their increasing demand on cognitive control) that precede any voluntary decision about how to "allocate" attention to one or more tasks.
} 


\section{References}

Alexander, N. B. (1994). Postural control in older adults. Journal of the American Geriatrics Society, 42, 93-108.

Anderson, P. G., Nienhuis, B., Mulder, T., \& Hulstijn, W. (1998). Are older adults more dependent on visual information in regulating selfmotion than younger adults? Journal of Motor Behavior, 30, 104-113.

Anstey, K. J., Lord, S. R., \& Williams, P. (1997). Strength in the lower limbs, visual contrast sensitivity, and simple reaction time cognition in older women. Psychology and Aging, 12, 137-144.

Anstey, K. J., Stankov, L., \& Lord, S. R. (1993). Primary aging, secondary aging, and intelligence. Psychology and Aging, 8, 562-570.

Baddeley, A., Della Sala, S., Gray, C., Papagno, C., \& Spinnler, H. (1997). Testing central executive functioning with a pencil-and-paper test. In P. M. A. Rabbitt (Ed.), Methodology of frontal and executive functions (pp. 61-80). Hove, England: Psychology Press.

Baddeley, A. D., \& Lieberman, K. (1980). Spatial working memory. In R. S. Nickerson (Ed.), Attention and performance (Vol. 3, pp. 521-539). Hillsdale, NJ: Erlbaum.

Baltes, P. B. (1987). Theoretical propositions of life-span developmental psychology: On the dynamics between growth and decline. Developmental Psychology, 23, 611-626.

Baltes, P. B. (1997). On the incomplete architecture of buman ontogeny. Selection, optimization, and compensation as foundation of developmental theory. American Psychologist, 52, 366-380.

Baltes, P. B., \& Kliegl, R. (1992). Further testing of limits of cognitive plasticity: Negative age differences in a mnemonic skill are robust. Developmental Psychology, 28, 121-125.

Baltes, P. B., \& Lindenberger, U. (1997). Emergence of a powerful connection between sensory and cognitive functions across the life span: A new window to the study of cognitive aging? Psychology and Aging, 12, 12-21.

Blake, A. J., Morgan, K., Bendall, M. J., Dallosso, H., Ebrahim, S. B. J., Arie, T. H. D., Fentem, P. H., \& Bassey, E. J. (1988). Falls by elderly people at home: Prevalence and associated factors. Age and Ageing, 17, 365-372.

Bower, G. H. (1970). Analysis of a mnemonic device. American Scientist, $58,496-510$.

Brinley, J. F. (1965). Cognitive sets, speed and accuracy of performance in the elderly. In A. T. Welford \& J. E. Birren (Eds.), Behavior, aging, and the nervous system (pp. 114-149). Springfield, IL: Charles C. Thomas.

Brown, L. A., Shumway-Cook, A., \& Woollacott, M. H. (1999). Attentional demands and postural recovery: The effects of aging. Journal of Gerontology: Medical Sciences, 54A, M165-M171.

Brown, L. A., \& Woollacott, M. H. (1998). The effects of aging on the control of posture and locomotion in healthy older adults: An emphasis on cognition. Psychologische Beiträge, 40, 27-43.

Bugelski, B. R. (1970). Words and things and images. American Psychologist, 25, 1002-1012.

Chen, H.-C., Ashton-Miller, J. A., Alexander, N. B., \& Schultz, A. B. (1994). Effects of age and available response time on ability to step over an obstacle. Journal of Gerontology: Medical Sciences, 49A, M227M233.

Chen, H.-C., Schultz, A. B., Ashton-Miller, J. A., Giordani, B., Alexander, N. B., \& Guire, K. E. (1996). Stepping over obstacles: Dividing attention impairs performance of old more than young adults. Joumal of Gerontology: Medical Sciences, 51A, M116-M122.

Craik, F. I. M., \& Byrd, M. (1982). Aging and cognitive deficits: The role of attentional resources. In F. I. M. Craik \& S. Trehub (Eds.), Aging and cognitive pracesses (pp. 191-211). New York: Plenum.

Craik, F. I. M., \& Jennings, J. M. (1992). Human memory. In F. I. M. Craik \& T. A. Salthouse (Eds.), The handbook of aging and cognition (pp. 51-110). Hillsdale, NJ: Erlbaum.

Duncan, J., Emslie, H., \& Williarns, P. (1996). Intelligence and the frontal lobes: The organization of goal-directed behavior. Cognitive Psychology, 30, 257-303.

Ericsson, K. A., \& Simon, H. A. (1993). Protocol analysis: Verbal reports as data (Rev. ed.). Cambridge, MA: MIT Press.

Grünbaum, B., \& Shephard, G. C. (1987). Tilings and patterns. New York: Freeman.

Hartley, A. A. (1992). Attention. In F. I. M. Craik \& T. A. Salthouse (Eds.), The handbook of aging and cognition (pp. 3-49). Hillsdale, NJ: Erlbaum.

Horn, J. L. (1989). Models of intelligence. In R. L. Linn (Ed.), Intelligence: Measurement, theory, and public policy (pp. 29-73). Urbana: University of Illinois Press.

Jopp, D. (1996). Erfassung von generellen, intellektuellen und auf intellektuelles Altem bezogenen Selbstwirksamkeitserwartungen [Assessment of general, intellectual, and intellectual-aging related self-efficacy beliefs]. Unpublished master's thesis, Free University of Berlin and Max Planck Institute for Human Development, Berlin, Germany.

Kerr, B., Condon, S. M., \& McDonald, L. A. (1985). Cognitive spatial processing and the regulation of posture. Journal of Experimental Psychology: Human Perception and Performance, 11, 617-622.

Kinsbourne, M., \& Hicks, R. E. (1978). Functional cerebral space: A model for overflow, transfer, and interference effects in human performance. In J. Requin (Ed.), Attention and performance (Vol. 7, pp. 345-362). Hillsdale, NJ: Erlbaum.

Klieg1, R., \& Lindenberger, U. (1993). Modeling intrusions and correct recall in episodic memory: Adult age differences in encoding of list context. Journal of Experimental Psychology: Leaming, Memory, and Cognition, 19, 617-637.

Kliegl, R., Mayr, U., \& Krampe, R. T. (1994). Time-accuracy functions for determining process and person differences: An application to cognitive aging. Cognitive Psychology, 26, 134-164.

Kliegl, R., Smith, J., \& Baltes, P. B. (1989). Testing-the-limits and the study of age differences in cognitive plasticity of a mnemonic skill. Developmental Psychology, 25, 247-256.

Korteling, J. E. (1993). Effects of age and task similarity on dual-task performance. Human Factors, 35, 99-113.

Kramer, A. F., Hahn, S., \& Gopher, D. (1999). Task coordination and aging: Explorations of executive control processes in the task switching paradigm. Acta Psychologica, 101, 339-378.

Kramer, A. F., Larish, J. F., \& Strayer, D. L. (1995). Training for attentional control in dual task settings: A comparison of young and old adults. Journal of Experimental Psychology: Applied, 1, 50-76.

Kray, J., \& Lindenberger, U. (2000). Adult age differences in task switching. Psychology and Aging, 15, 126-147.

Lajoie, Y., Teasdale, N., Bard, C., \& Fleury, M. (1996). Attentional demands for walking: Age-related changes. In A.-M. Ferrandez \& N. Teasdale (Eds.), Changes in sensory motor behavior in aging (pp. 235-256). Amsterdam: Elsevier.

Lea, G. (1975). Chronometric analysis of the method of loci. Journal of Experimental Psychology: Human Perception and Performance, 104 95-104.

Li, K. Z. H., Lindenberger, U., Freund, A., \& Baltes, P. B. (2000). Walking while memorizing: An SOC study of age-related differences in compensatory behavior under dual-task conditions. Unpublished manuscript.

Light, L. L. (1996). Memory and aging. In E. L. Bjork \& R. A. Bjork (Eds.), Memory (pp. 444-490). San Diego, CA: Academic Press.

Lindenberger, U., \& Baltes, P. B. (1994). Sensory functioning and intelligence in old age: A strong connection. Psychology and Aging, 9, 339-355.

Lindenberger, U., \& Baltes, P. B. (1997). Intellectual functioning in old and very old age: Cross-sectional results from the Berlin Aging Study. Psychology and Aging, 12, 410-432.

Lindenberger, U., Kliegl, R., \& Baltes, P. B. (1992). Professional expertise 
does not eliminate age differences in imagery-based memory performance during adulthood. Psychology and Aging, 7, 585-593.

Lindenberger, U., Mayr, U., \& Kliegl, R. (1993). Speed and intelligence in old age. Psychology and Aging, 8, 207-220.

Lundin-Olsson, L., Nyberg, L., \& Gustafson, Y. (1997). Attention, frailty, and falls: The effect of a manual task on basic mobility. Joumal of the American Geriatric Society, 46, 758-761.

Lundin-Olsson, L., Nyberg, L., \& Gustafson, Y. (1998). "Stops when talking" as a predictor of falls in elderly people. The Lancet, 349, 617.

Marsiske, M., Klumb, P., \& Baltes, M. M. (1997). Everyday activity patterns and sensory functioning in old age. Psychology and Aging, 12, 444-457.

Maylor, E. A., Allison, S., \& Wing, A. M. (in press). Effects of spatial and nonspatial cognitive activity on postural stability. British Journal of Psychology.

Maylor, E. A., \& Wing, A. M. (1996). Age differences in postural stability are increased by additional cognitive demands. Journal of Gerontology: Psychological Sciences, 51B, P143-P154.

McDowd, J. M., \& Craik, F. I. M. (1987). Effects of aging and task difficulty on divided attention performance. Journal of Experimental Psychology: Human Perception and Performance, 14, 267-280.

McDowd, J. M., \& Shaw, R. (2000). Attention and aging: A functional perspective. In F. I. M. Craik \& T. A. Salthouse (Eds.), Handbook of aging and cognition (2nd ed., pp. 221-292). Mahwah, NJ: Erlbaum.

Mulder, T., Berndt, H., Pauwels, J., \& Nienhuis, B. (1993). Sensorimotor adaptability in the elderly and the disabled. In G. E. Stelmach \& V. Hömberg (Eds.), Sensorimotor impairment in the elderly (pp. 413-426). Dordrecht, The Netherlands: Kluwer Academic.

Navon, D., \& Gopher, D. (1979). On the economy of the human-processing system. Psychological Review, 80, 214-255.

Norman, D. A., \& Bobrow, D. G. (1975). On data-limited and resourcelimited processes. Cognitive Psychology, 7, 44-64.

Raven, J. C., Court, J. H., \& Raven, J. (1983). Manual for Raven's progressive matrices and vocabulary scales: Advanced Progressive Matrices Sets I and II. London: H. K. Lewis.

Ribaupierre, A. de, \& Ludwig, C. (2000). Aftention divisée et viellissement cognitif: Différences d'âge dans 5 épreuves duelles de mémoire de travail [Divided attention and cognitive aging: Age differences in five working memory dual tasks]. In D. Brouillet (Ed.), Le vieillissement cognitif normal. Vers un modèle explicatif du vieillissement [Normal cognitive aging. Towards an explanatory model] (pp. 29-51). Brussels, Belgium: DeBoek.

Richardson, J. T. E. (1996). Measures of effect size. Behavior Research Methods, Instruments, and Computers, 28, 12-22.

Salthouse, T. A. (1988). The complexity of age by complexity functions: Comment on Charness and Campbell (1988). Journal of Experimental Psychology: General, 117, 425-428.

Salthouse, T. A., Rogan, D. J., \& Prill, K. A. (1984). Division of attention: Age differences on a visually presented memory task. Memory \& Cognition, 12, 613-620.

Salthouse, T. A., Hambrick, D. Z., Lukas, K. E., \& Dell, T. C. (1996). Determinants of adult age differences on synthetic work performance. Journal of Experimental Psychology: Applied, 2, 305-329.

Shallice, T., \& Burgess, P. (1993). Supervisory control of action and thought selection. In A. Baddeley \& L. Weiskrantz (Eds.), Attention: Selection, awareness, and control (pp. 171-187). New York: Oxford University Press.

Shumway-Cook, A., Woollacott, M., Kerns, K. A., \& Baldwin, M. (1997).
The effects of two types of cognitive tasks on postural stability in older adults with and without a history of falls. Journal of Gerontology: Medical Sciences, 52A, M232-M240.

Spengler, F., Godde, B., \& Dinse, H. R. (1995). Effects of ageing on topographic organization of somatosensory cortex. NeuroReport, 6 , $469-473$.

Stelmach, G. E., Teasdale, N., DiFabio, R. P., \& Phillips, J. (1989). Age-related decline in postural control mechanisms. International Journal of Aging and Human Development, 29, 205-223.

Stelmach, G. E., Zelaznik, H. N., \& Lowe, D. (1990). The influence of aging and attentional demands on recovery from postural instability. Clinical and Experimental Aging, 2, 155-161.

Stigsdotter Neely, A., \& Bäckman, L. (1993). Long-term maintenance of gains from memory training in older adults: Two $31 / 2$ years follow-up studies. Journal of Gerontology: Psychological Sciences, 5, 233-237.

Teasdale, N., Bard, C., Dadouchi, F., Fleury, M., LaRue, J., \& Stelmach, G. E. (1992). Posture and elderly persons: Evidence for deficits in the central integrative mechanisms. In G. E. Stelmach \& J. Requin (Eds.), Tutorials in motor behavior (Vol. 2, pp. 917-931). Amsterdam: NorthHolland.

Teasdale, N., Bard, C., LaRue, J., \& Fleury, M. (1993). On the cognitive penetrability of posture control. Experimental Aging Research, 19, 1-13.

Teasdale, N., Lajoie, Y., Bard, C., Fleury, M., \& Courtemanche, R. (1993). Cognitive processes involved for maintaining postural stability while standing and walking. In G. E. Stelmach \& V. Hömberg (Eds.), Sensorimotor impairment in the elderly (pp. 157-168). Dordrecht, The Netherlands: Kluwer.

Teasdale, N., Stelmach, G. E., \& Breunig, A. (1991). Postural sway characteristics of elderly persons under normal and altered visual and support surface conditions. Joumal of Gerontology: Biological Sciences, 46, B238-B244.

Tsang, P. S., \& Shaner, T. L. (1998). Age, attention, expertise, and time-sharing perfornance. Psychology and Aging, 13, 323-347.

Wechsler, D. (1982). Handanweisung zum Hamburg-Wechsler-Intelligenztest für Erwachsene (HAWIE) [Manual for the Hamburg-Wechsler Intelligence Test for Adults]. Bern, Switzerland: Huber.

Welford, A. T. (1958). Ageing and human skill. London: Oxford University Press.

Wickens, C. D. (1984). Processing resources in attention. In R. Parasuraman \& D. R. Davies (Eds.), Varieties of attention (pp. 63-98). New York: Academic Press.

Winocur, G., \& Moscovitch, M. (1983). Paired-associate learning in institutionalized and noninstitutionalized old people: An analysis of interference and context effects. Journal of Gerontology, 38, 455-464.

Winter, D. A. (1991). The biomechanics and motor control of human gait: Normal, elderiy, and pathological (2nd ed.). Waterloo, Ontario, Canada: University of Waterloo Press.

Woollacott, M. H., \& Jensen, J. L. (1996). Posture and locomotion. In H. Heuer \& S. W. Keele (Eds.), Handbook of perception and action: Vol. 2. Motor skills (pp. 333-403). London: Academic Press.

Zacks, R. T., Hasher, L., \& Li, K. Z. H. (2000). Human memory. In F. I. M. Craik \& T. A. Salthouse (Eds.), Handbook of aging and cognition (2nd ed., pp. 283-357). Mahwah, NJ: Erlbaum.

Received July 30, 1999

Revision received November 23, 1999 Accepted November 28, 1999 